\title{
Algebraic and Topological Aspects of Feedback Stabilization
}

\author{
MATHUKUMALLI VIDYASAGAR, SENIOR MEMBER, IEEE, HANS SCHNEIDER, AND \\ BRUCE A. FRANCIS, MEMBER, IEEE
}

\begin{abstract}
In this paper we give essentially complete results concerning various algebraic and topological aspects of feedback stabilization. In particular, we give necessary and sufficient conditions for a given transfer function matrix to have a right-coprime or a left-coprime factorization, and exhibit a large class of transfer function matrices that have both. We give the most general set of feedback stability criteria available to date, and derive a characterization of all compensators that stabilize a given plant. We give a definition of "proper" and "strictly proper" in an abstract setting and show that 1 ) every strictly proper plant can be stabilized by a proper compensator, and 2) every compensator that stabilizes a strictly proper plant must be proper. We then define a topology for unstable plants and compensators, and show that it is the weakest topology in which feedback stability is a robust property.
\end{abstract}

\section{INTRODUCTION}

$I^{\prime}$ N THIS PAPER we study various algebraic and topological aspects of feedback stabilization. We develop general results in answer to the following types of questions. Given an unstable plant, can one find a feedback compensator to stabilize it? Can one characterize all feedback compensators that stabilize a given plant, stable or unstable? If the plant is known to be strictly proper, can one achieve stabilization using a proper compensator? What sort of uncertainty can be tolerated in the plant model and in the implementation of the compensator? What is meant by an unstable system being "close" to another?

Our starting point is the observation that in a wide variety of applications the set of all single-input-singleoutput (SISO) stable linear systems forms a ring $\mathcal{K}$; that is, parallel and cascade connections of stable linear systems are again stable linear systems. Moreover, in many cases (e.g., convolution operators) the ring $\mathcal{H}$ is commutative and is an integral domain (i.e., $\mathcal{H}$ has no divisors of zero). ${ }^{\prime}$ One can study multiinput-multioutput (MIMO) stable linear systems by viewing them as matrices over the ring $\mathcal{H}$. The class of SISO unstable systems considered in this paper are the elements of the quotient field $\mathscr{F}$ of $\mathcal{K}$. Note that if

Manuscript received April 9, 1980; revised September 25, 1980, September 4, 1981, and January 4, 1982. Paper recommended by A. J. Laub, Past Chairman of the Computational Methods and Discrete Systems Committee. This work was supported by the Natural Sciences and Engineering Research Council of Canada under Grant A-1240 (M:V.) and the National Science Foundation under Grants MCS-78-01087 (H.S.) and ECS-80-12565 (B.A.F.).

M. Vidyasagar and B. A. Francis are with the Department of Electrical Engineering, University of Waterloo, Waterloo, Ont., Canada N2L 3GI.

$\mathrm{H}$. Schneider is with the Department of Mathematics, University of Wisconsin, Madison, WI 53706.

'See Jacobson [1] for definitions of terminology from ring theory. $g \in \mathscr{F}$, then $g d \in \mathcal{H}$ for some nonzero $d \in \mathcal{H}$. Hence, the SISO unstable systems considered here are those that can be stabilized by the cascade connection of some nontrivial stable system. We treat the MIMO case by considering matrices over $\mathscr{F}$.

The advantage of using such a setup is that it embraces, within a single framework, continuous-time as well as discrete-time systems, lumped as well as distributed systems, $1-D$ as well as $n$-D systems, etc. As a result, when we study problems such as feedback stabilization, we are able to focus on the key aspects of the problem under study, rather than be distracted by the special features of a particular class of linear systems. In spite of the fact that we use terminology from ring theory in the statement and the proofs of the results given here, we do not use any deep results from ring theory. The background needed to follow the paper is all contained in [1, ch. II-IV]. In particular, the reader would need to know what a ring is, and what is meant by ideal, principal ideal, and prime ideal in a ring.

There are three types of problems considered in this paper. First, a central idea in studying feedback stability is that of expressing an unstable transfer function $g$ as a ratio of two stable transfer functions $n / d$ in such a way that $n$ and $d$ are coprime [2], [3]. In this connection an important question is the following. Given a transfer function $g=$ $n_{1} / d_{1}$ where $n_{1}$ and $d_{1}$ are not necessarily coprime; when is it possible to find a coprime pair $(n, d)$ such that $n / d=$ $n_{1} / d_{1}$ ? In the practically important case where $g$ is $\tilde{a}$ rational function of $s$, this is always possible [4], [5]; however, in the study of linear distributed systems where $g$ may be an irrational function of $s$, this is not always possible (see Example 2.43). In Section II of this paper we present several results that show just when it is possible to find a coprime factorization of a given ratio $n_{1} / d_{1}$ (or $N_{1} D_{1}^{-1}$ in the MIMO case). These results contain some earlier work in [6], [7] as special cases.

We then consider the problem of designing a feedback compensator that stabilizes a given plant. This problem can be split into two parts: 1) finding necessary and sufficient conditions for a feedback system to be stable, and characterizing the collection of all compensators that stabilize a given plant, and 2) determining whether or not a given plant can be stabilized (i.e., whether or not the "set of stabilizing compensators" is nonempty), and determining whether a "strictly proper" plant can be stabilized by a "proper" compensator. These problems are discussed in 
Section III. Specifically, we derive the most general set of necessary and sufficient conditions for feedback stability available to date, and we give a complete characterization of all compensators that stabilize a given plant. These results extend those in [8] by removing several technical assumptions, and can be thought of as an abstract version of [9]. Next, we give a definition of a "proper" and "strictly proper" element in an abstract ring-theoretic setting and prove two important results: 1) every strictly proper plant can be stabilized by a proper compensator, and 2) every compensator that stabilizes a strictly proper plant must be proper. These results not only generalize those of [10], [11], but are also proved in a considerably simpler manner than the special cases in [10], [11].

The final issue studied in this paper is that of defining a suitable topology in which feedback stability is robust. This is of fundamental importance since a controller designed for a nominal plant must accommodate uncertainty about the nominal plant model and in the controller implementation. In other words, the feedback system must continue to be stable for all plants in some neighborhood of the nominal plant and for all controllers in some neighborhood of the nominal controller. Topologizing the plants and controllers means defining these neighborhoods. In Section IV we define a topology for unstable plants and show that it is the weakest topology in which closed-loop stability is a robust property. The principle that emerges from this section is that in order to design a controller for a plant that can also accommodate plant uncertainty, we must know approximately the stable coprime factors of the plant.

Throughout the paper we use script letters to denote sets and Roman letters to denote elements of sets. Also, symbols with a " " denote quantities pertaining to leftcoprime factorizations, whereas quantities pertaining to right-coprime factorizations do not have the tilde.

\section{COPRIME FACTORIZATIONS}

\section{A. Basic Concepts}

Throughout this section we let ${ }^{2}$

$\mathcal{K}=$ a commutative ring with identity and no zero divisors,

$\mathcal{G}=$ set of multiplicative units of $\mathcal{K}=\{x \in \mathcal{K}: x$ has a multiplicative inverse in $\mathcal{H}$,

$\mathscr{F}=$ quotient field of $\mathscr{K}=\{a / b: a \in \mathcal{K}, b \in \mathcal{K} \backslash 0\}$.

Since many engineering systems are multiinput-multioutput, we introduce the set $\mathcal{K}^{n \times m}$, which is the set of $n \times m$ matrices whose elements all belong to $\mathcal{K}$. Similarly, $\mathscr{F}^{n \times m}$ denotes the set of $n \times m$ matrices whose elements all belong to $\mathscr{F}$. It is easy to see that both $\mathcal{H}^{n \times n}$ and $\mathscr{F}^{n \times n}$ are rings under the usual definitions of addition and multiplication, and are noncommutative if $n \geqslant 2$. We can also speak of the determinant of an element of $\mathcal{K}^{n \times n}$ and $\mathscr{F}^{n \times n}$,

${ }^{2}$ See $[1, \mathrm{p} .49]$ for a definition of these terms. defined in the usual way. It is easy to see that every element $F$ of $\mathscr{F}^{n \times m}$ can be written as $N D^{-1}$, where $N \in$ $\mathcal{K}^{n \times m}, D \in \mathcal{H}^{m \times m}$ and det $D \neq 0$; we refer to such a pair $(N, D)$ as a right fractional representation of $F$. Similarly, every $F \in \mathscr{F}^{n \times m}$ can also be written as $\tilde{D}^{-1} \tilde{N}$, where $\tilde{D} \in$ $\mathcal{K}^{n \times n}, \tilde{N} \in \mathcal{H}^{n \times m}$ and $\operatorname{det} \tilde{D} \neq 0$; such a pair $(\tilde{D}, \tilde{N})$ is referred to as a left fractional representation of $F^{3}$

Note that the set of $m$-tuples $\mathcal{F}^{m}$ is a module [27, p. 134] over the ring $\mathcal{K}$. Thus, in the present setup $\mathcal{H}^{n \times m}$ is the set of "stable transfer matrices" that map the input module $\mathcal{H}^{m}$ into the output module $\mathcal{F}^{n}$. $\mathscr{F}^{n \times m}$ plays the role of all possible transfer matrices, both stable as well as unstable.

We say that two matrices $N \in \mathcal{H}^{n \times m}, D \in \mathcal{H}^{1 \times m}$ are right-coprime if there exist matrices $P \in \mathcal{H}^{m \times n}, Q \in \mathcal{H}^{m \times 1}$ such that

$$
P N+Q D=I_{m}
$$

where $I_{m}$ denotes the identity matrix of dimension $m$. Note that the present definition of coprimeness implies not only that every common right divisor of $N$ and $D$ is a unit in $\mathcal{H}^{m \times m}$, but also that every common right divisor of $N$ and $D$ is contained in the ideal generated by $N$ and $D$. If $\mathcal{H}$ is a Bezout domain (i.e., if every finitely generated ideal in $\mathcal{K}$ is principal), then the first condition can be shown to imply the second (see, e.g., [28]).

Suppose $F \in \mathscr{F}^{n \times m}$; then $(N, D)$ is a right-coprime factorization ( $r c f$ ) of $F$ if 1$)$ det $D \neq 0$ and $F=N D^{-1}$, and 2) $N$ and $D$ are right coprime. Left coprimeness and left coprime factorizations (lcf's) are defined analogously.

It is well known $[8, \mathrm{p} .401$, Property 2] that if $(N, D)$ is an rcf of $F \in \mathscr{F}^{n \times m}$, then so is $(N U, D U)$ where $U \in \mathcal{H}^{m \times m}$ is a unimodular matrix (i.e., $\operatorname{det} U \in g$ ). ${ }^{4}$ Moreover, if $\left(N_{1}, D_{1}\right)$ is another rcf of $F$, there exists a unimodular matrix $V \in \mathcal{H}^{m \times m}$ such that $N_{1}=N V, D_{1}=D V$. Thus, an rcf of an element in $\mathscr{F}^{n \times m}$ is unique to within a right associate if an rcf exists. In the case of lumped linear systems, for example, the existence of coprime factorizations is not a problem because $\mathcal{H}$ is a principal ideal domain in such applications. However, in the study of distributed systems, multidimensional systems, etc., the existence of coprime factorizations is not automatic. As a matter of fact, in both these applications not every element of $\mathcal{F}$ has a coprime factorization, as demonstrated in Examples 2.1 and 2.2. To pave the way for these examples, we present a lemma and two corollaries, the proofs of which can be found in [28].

Lemma 2.1: Suppose $F \in \mathscr{F}^{n \times m}$, and let $(N, D)$ be any right fractional representation of $F$. Then $F$ has an rcf if and only if the left ideal in $\mathcal{H}^{m \times m}$ generated by $N$ and $D$ is a left principal ideal.

\footnotetext{
${ }^{3}$ In [8] Desoer et al. introduce the concept of a "fractional representation" and derive several useful results. One of the assumptions made in $[8]$ is that every "right fraction" $N D^{-1}$ also has a "left fractional representation" of the form $\tilde{D}^{-1} \bar{N}$. In the current setup we start with a commutative "scalar ring" $\mathcal{K}$ and build up the matrix rings $\mathcal{K}^{\pi \times m}$ and $\sigma^{n \times m}$; as a result, this assumption is automatically satisfied, in the current setting.

${ }^{4}$ Recall that $g$ is the set of multiplicative units of $\mathcal{K}$
} 
Corollary 2.1: Suppose $f \in \mathscr{F}$, and $f=n / d$ where $n \in \mathcal{H}$ and $d \in \mathscr{H} \backslash 0$. Then $f$ has a coprime factorization ${ }^{5}$ if and only if the ideal in $\mathcal{K}$ generated by $n$ and $d$ is principal.

Corollary 2.2: Every $f \in \mathcal{F}$ has a coprime factorization if and only if $\mathcal{H}$ is a Bezout domain.

\section{B. Examples}

In this section we discuss three rings that are commonly encountered in stability theory, and show that they are not Bezout domains, so that not every element in the quotient field associated with these rings has a coprime factorization.

Example 2.1: In the case of discrete-time systems it is well known [15] that a linear time-invariant system is BIBO stable if and only if its impulse response is absolutely summable. Hence, a natural choice for $\mathcal{H}$ is $l_{1}$, the set of all absolutely summable sequences. For ease of multiplication it is better to take

$$
\mathcal{H}=\left\{\tilde{f}(z)=\sum_{i=0}^{\infty} f_{1} z^{i}, \sum_{i=0}^{\infty}\left|f_{i}\right|<\infty\right\} .
$$

In other words, we take $\mathcal{H}$ to be the set of power series with absolutely summable coefficients, which is isomorphic to $l_{1}$. Note that $\tilde{f}\left(z^{-1}\right)$ is the usual $z$ transform of the sequence $\left\{f_{i}\right\}$.

The ring $\mathcal{H}$ does not have the property that every finitely generated ideal is principal, as the following argument shows. Hence, not every element of the quotient field of $\mathcal{H C}$ has a coprime factorization.

First, suppose $\left\{\alpha_{i}\right\}$ is a sequence of real numbers converging to 1 such that $\left|\alpha_{i}\right|<1$ for all $i$, and such that the sum $\sum_{i=0}^{\infty}\left(1-\alpha_{i}^{4}\right)$ is finite. Then, we claim that there exists a sequence $\left\{f_{n}\right\}$ in $l_{1}$ such that $\tilde{f}\left(\alpha_{i}\right)=0$ for all $i$ and $f(z) \neq 0$ elsewhere. ${ }^{6}$ In fact, let

$$
\tilde{f}(z)=\tilde{g}(z) \exp \left[-1 /(1-z)^{1 / 2}\right]
$$

where $\tilde{g}(\cdot)$ is the Blaschke product $[29$, p. 66]

$$
\tilde{g}(z)=\prod_{i=0}^{\infty} \frac{z-\alpha_{i}}{1-\alpha_{i} z}
$$

Then $|\tilde{g}(z)| \leqslant 1$ whenever $|z| \leqslant 1$, and $\tilde{g}(\cdot)$ is analytic over the open unit disk. Moreover, $\tilde{g}(\cdot)$ is continuous everywhere on the unit circle except at $z=1$. Now, because of the exponential term in (2.3), $\tilde{f}(\cdot)$ is continuous at $z=1$, and $\tilde{f}(1)=0$. Moreover, the exponential term in (2.3) approaches zero as $z \rightarrow 1$ faster than any term of the form $1 /(z-1)^{n}$ for any $n>0$. With the aid of this and the convergence of $\sum_{i=0}^{\infty}\left(1-\alpha_{i}^{4}\right)$, one can verify that $\tilde{f}(\cdot)$ is twice continuously differentiable on the unit circle, including at $z=1$. Consider now the harmonic series

\footnotetext{
${ }^{5}$ Since $\mathscr{F}$ is commutative we need not distinguish between rcf's and lcf's.

${ }^{6}$ We are grateful to $\mathrm{K}$. Davidson for this argument.
}

$$
\tilde{f}\left(e^{j \theta}\right)=\sum_{n=0}^{\infty} f_{n} e^{j n \theta}
$$

Since $\tilde{f}\left(z^{*}\right)=\tilde{f}^{*}(z)$ where ${ }^{*}$ denotes conjugation, it follows that $f_{n}$ is real for all $n$. Also, since $\tilde{f}(\cdot)$ is $C^{2}, f_{n}=0\left(1 / n^{2}\right)$ and hence $\left\{f_{n}\right\} \in l_{1}=\mathcal{H}$.

Now we show that $\mathcal{H}$ is not a Bezout domain. Let $\left\{\alpha_{i}\right\}$ $\left\{\beta_{i}\right\}$ be two sequences in the open unit disk such that 1) $\alpha_{i}, \beta_{i}$ are real for all $\left.\left.i, 2\right) \alpha_{i} \rightarrow 1, \beta_{i} \rightarrow 1,3\right) \sum_{i=0}^{\infty}\left(1-\alpha_{i}^{4}\right)<\infty$, $\sum_{i=0}^{\infty}\left(1-\beta_{i}^{4}\right)<\infty$, and 4) $\alpha_{i} \neq \beta_{j}$ for all $i, j .^{7}$ As shown above, we can find $l_{1}$ sequences $\left\{f_{n}\right\},\left\{g_{n}\right\}$ such that $\left\{\alpha_{i}\right\}$ is the zero set of $\tilde{f}(\cdot),\left\{\beta_{i}\right\}$ is the zero set of $\tilde{g}(\cdot)$. We show that $f$ and $g$ do not generate a principal ideal in $\mathcal{H} .^{8}$ To show this, assume the contrary; namely, that there exist $\tilde{h}_{1}, \cdots, \tilde{h}_{5}$ in $\mathcal{H}$ such that

$$
\begin{gathered}
\tilde{h}_{1}(z) \tilde{f}(z)+\tilde{h}_{2}(s) \tilde{g}(z)=\tilde{h}_{3}(z), \quad \text { if }|z| \leqslant 1 \quad(2.6) \\
\tilde{f}(z)=\tilde{h}_{3}(z) \tilde{h}_{4}(z), \quad \tilde{g}(z)=\tilde{h}_{3}(z) \tilde{h}_{5}(z), \quad \text { if }|z| \leqslant 1 .
\end{gathered}
$$

Then $\tilde{h}_{4} \tilde{g}=\tilde{h_{5}} \tilde{f}$, so that $\tilde{h}_{4}\left(\alpha_{i}\right)=0, \tilde{h}_{5}\left(\beta_{i}\right)=0, \forall i$. Also, since

$$
\tilde{h}_{1}(z) \tilde{h}_{4}(z)+\tilde{h}_{2}(z) \tilde{h}_{5}(z)=1 \quad \text { if }|z| \leqslant 1
$$

we have

$$
\tilde{h}_{1}\left(\beta_{i}\right) \tilde{h}_{4}\left(\beta_{i}\right)=1 \quad \forall i .
$$

If we let $i \rightarrow \infty$ in (2.9), we get $\tilde{h}_{1}(1) \tilde{h}_{4}(1)=1$. However, $\tilde{h}_{4}(1)=\lim _{i \rightarrow \infty} \tilde{h}_{4}\left(\alpha_{i}\right)=0$, which is a contradiction. Hence, $\tilde{f}$ and $\tilde{g}$ do not generate a principal ideal, and $\mathcal{H}=l_{1}$ is not a Bezout domain. As a result, not every element of the quotient field of $\mathscr{K}$ has a coprime factorization.

Example 2.2: In the study of continuous-time systems, a natural choice for $\mathcal{H}$ is the Banach algebra $\mathcal{Q}$ defined in [13]. The set $\mathcal{Q}$ consists of all distributions $f(\cdot)$ of the form

$$
f(t)=\left\{\begin{array}{l}
0, \quad t<0 \\
f_{a}(t)+\sum_{i=0}^{\infty} f_{i} \delta\left(t-t_{i}\right), \quad t \geqslant 0
\end{array}\right.
$$

where $0 \leqslant t_{0}<t_{1}<\cdots, f_{a}(\cdot) \in L_{1}$ and $\left\{f_{i}\right\} \in l_{1}$. If we define the norm of an element $f \in \mathbb{Q}$ as

$$
\|f(\cdot)\|_{\mathscr{Q}}=\int_{0}^{\infty}\left|f_{a}(t)\right| d t+\sum_{i=0}^{\infty}\left|f_{i}\right|
$$

and define the product of two elements as their convolution, then $\mathbb{Q}$ is a Banach algebra and satisfies all of the hypotheses we have imposed on $\mathcal{K}$. Also, by the PaleyWiener theorem $[14$, p. 150], the set of units of $\mathcal{Q}$ is

$$
g=\left\{f \in \mathbb{Q}: \inf _{s \in C_{+}}|\hat{f}(s)|>0\right\}
$$

${ }^{7}$ Since $\left(1-\alpha^{4}\right)=(1-\alpha)\left(1+\alpha+\alpha^{2}+\alpha^{3}\right) \leqslant 4(1-\alpha)$ whenever $0 \leqslant \alpha \leqslant$ $1,(3)$ is equivalent to $\sum_{i=0}^{\infty}\left(1-\alpha_{i}\right)<\infty$.

${ }^{8} \mathrm{We}$ are grateful to $W$. Rudin for this argument 
where $\hat{f}(\cdot)$ denotes the Laplace transform of $f(\cdot)$ and $C_{+}=\{s: \operatorname{Re} s \geqslant 0\}$. In other words, $f \in \mathbb{Q}$ has an inverse in $\mathbb{Q}$ if and only if its Laplace transform is bounded away from zero over $C_{+}$.

Now the set of distributions of the form

$$
f(t)=\sum_{i=0}^{\infty} f_{i} \delta(t-i T), \quad\left\{f_{i}\right\} \in l_{1}, \quad T>0
$$

is a subalgebra of $\mathbb{Q}$ which is isomorphic to $l_{1}$. As we have already shown in Example 2.1, $l_{1}$ is not a Bezout domain. It is easy to adapt these arguments to show that $\mathbb{Q}$ is not a Bezout domain. Hence, not every element in the quotient field of $\mathbb{Q}$ has a coprime factorization.

Example 2.3: In the study of two-dimensional digital filters, a natural choice for $\mathcal{H}$ is the set of real-valued sequences $\left\{f_{i j}\right\}$ such that

$$
\sum_{i=0}^{\infty} \sum_{j=0}^{\infty}\left|f_{i j}\right|<\infty
$$

and such that the two-dimensional $z$ transform

$$
\tilde{f}\left(z_{1}, z_{2}\right)=\sum_{i=0}^{\infty} \sum_{j=0}^{\infty} f_{i j} z_{1}^{i} z_{2}^{j}
$$

is a rational function of $z_{1}, z_{2}$. Note that every two-variable polynomial with real coefficients belongs to $\mathcal{H}$. In other words, the ring $R\left[z_{1}, z_{2}\right]$ is a subset of $\mathcal{H}$.

Now consider the elements

$$
\tilde{f}\left(z_{1}, z_{2}\right)=z_{1}, \quad \tilde{g}\left(z_{1}, z_{2}\right)=z_{2} .
$$

One can use essentially the same arguments as in Example 2.1 to show that the ideal in $\mathcal{H}$ generated by $\tilde{f}$ and $\tilde{g}$ is not principal. Assume by way of contradiction that there exist $\tilde{f}_{1}, \tilde{g}_{1}, \tilde{a}, \tilde{b}, \tilde{h}$ in $\mathscr{K}$ such that

$$
\begin{gathered}
\tilde{f}=\tilde{f}_{1} \tilde{h}, \quad \tilde{g}=\tilde{g}_{1} \tilde{h} \\
\tilde{a} \tilde{f}+\tilde{b} \tilde{g}=\tilde{h} .
\end{gathered}
$$

Then $\tilde{f}_{1} \tilde{g}=\tilde{g}_{1} \tilde{f}$, so that $\tilde{f}_{1}\left(0, z_{2}\right)=0$ for all $z_{2} \neq 0, \tilde{g}_{1}\left(z_{1}, 0\right)$ $=0$ for all $z_{1} \neq 0$. Also, cancelling $\tilde{h}$ in $(2.18)$ gives

$$
\tilde{a}\left(z_{1}, z_{2}\right) \tilde{f}_{1}\left(z_{1}, z_{2}\right)+\tilde{b}\left(z_{1}, z_{2}\right) \tilde{g}_{1}\left(z_{1}, z_{2}\right)=1 \text {. }
$$

Letting $z_{1} \rightarrow 0, z_{2} \rightarrow 0$ in (2.19) gives the desired contradiction. Hence, $z_{1} / z_{2}$ has no coprime factorization.

\section{Coprime Factorizations}

As shown by the examples in Section II-B, several commonly encountered rings in stability theory are not Bezout domains. In such cases it is of interest to identify some subring $\mathcal{G}$ of $\mathscr{F}$ such that every $g \in \mathscr{F}$ has a coprime factorization, and more important every $G \in \mathcal{G}^{n \times m}$ has both an rcf as well as an lcf. Such a result is provided by Theorem 2.1 below. We first state a preliminary lemma.

Lemma 2.2: Suppose $N \in \mathcal{K}^{n \times m}, D \in \mathcal{K}^{m \times m}$, with det $D \neq 0$. If there exists a unimodular matrix $R \in$
$\mathcal{K}^{(n+m) \times(n+m)}$ such that

$$
R\left[\begin{array}{l}
D \\
N
\end{array}\right]=\left[\begin{array}{l}
T \\
0_{n \times m}
\end{array}\right]
$$

where $0_{n \times m}$ denotes the zero matrix of order $n \times m$, then $G=N D^{-1}$ has an rcf.

Proof: Partition $R$ as

$$
R=\left[\begin{array}{cc}
m & n \\
R_{11} & R_{12} \\
R_{21} & R_{22}
\end{array}\right] \begin{aligned}
& m \\
& n
\end{aligned}
$$

Now (2.20) implies that

$$
R=R_{11} D+R_{12} N .
$$

Since det $R \in \mathcal{F}, S \triangleq R^{-1}$ belongs to $\mathcal{K}^{(n+m) \times(n+m)}$. Partition $S$ as in (2.21). Then, since

$$
\left[\begin{array}{l}
D \\
N
\end{array}\right]=\left[\begin{array}{ll}
S_{11} & S_{12} \\
S_{21} & S_{22}
\end{array}\right]\left[\begin{array}{l}
T \\
0
\end{array}\right]
$$

it follows that

$$
D=S_{11} T, \quad N=S_{21} T .
$$

Since $\operatorname{det} D \neq 0$, it is clear that $\operatorname{det} T \neq 0$ and $\operatorname{det} S_{11} \neq 0$. Hence, $G=N D^{-1}=S_{21} S_{11}^{-1}$. Moreover, since $R S=I_{(n+m)}$,

$$
R_{12} S_{21}+R_{11} S_{11}=I_{n}
$$

which shows that $S_{21}, S_{11}$ are right-coprime. Hence, $\left(S_{21}, S_{11}\right)$ is an $\operatorname{ref}$ of $N D^{-1}$.

We now come to the main result.

Theorem 2.1: Given $\mathcal{K}$, suppose that there exists a subring $\mathcal{H}_{0}$ of $\mathcal{H}$ and a subset $\mathscr{G}$ of $\mathcal{H}_{0} \backslash 0$ such that

1) $\mathscr{G}$ is closed under multiplication (i.e., $x \in \mathscr{G}, y \in \mathscr{G}$ implies that $x y \in \mathscr{G}$ ).

2) Every factor in $\mathcal{H}_{0}$ of an element of $\mathscr{G}$ belongs to $q$ (i.e., $x \in \mathcal{H}_{0}, y \in \mathcal{H}_{0}, x y \in \mathscr{G}$ implies that $x \in \mathscr{G}, y \in \mathscr{G}$ ).

3) Whenever $x \in \mathcal{K}_{0}$ and $y \in \mathscr{G}$, the ideal in $\mathcal{H}_{0}$ generated by $x$ and $y$ is principal.

Under these conditions the set of fractions $\mathcal{G} \triangleq\{n / d$, $\left.n \in \mathcal{K}_{0}, d \in \mathscr{I}\right\}$ is a subring of $\mathscr{F}$. Moreover, for every $n, m$, every element of $\mathcal{G}^{n \times m}$ has both an rcf and an lcf.

\section{Remarks:}

1) In the above theorem it is important to note that the hypotheses pertain to the "scalar" ring $\mathcal{H}_{0}$ and its subset $q$, but the conclusions pertain to the matrix ring $\mathcal{G}^{n \times m}$.

2) Assumptions 1) and 2) imply that $\mathscr{G}$ is a saturated multiplicative system in $\mathcal{K}_{0}$ and that $\mathcal{G}$ is the corresponding local ring $\mathscr{G}^{-1} \mathcal{K}_{0}[28$, p. 38]. Note that these assumptions are automatically satisfied if $g$ is the complement of a prime ideal in $\mathscr{H}_{0}$.

3) Combining Assumptions 2) and 3), one can prove the following. Whenever $x_{1}, \cdots, x_{k} \in \mathcal{H}_{0}$ and $y \in \mathcal{G}$, the ideal in $\mathcal{H}_{0}$ generated by $x_{1}, \cdots, x_{k}, y$ is principal.

Proof: In view of Assumption 1) it is immediate that $\mathcal{G}$ is a subring of $\widetilde{F}$.

To prove that every element of $\mathcal{G}^{n \times m}$ has an rcf, let $G \in \mathcal{G}^{n \times m}$. Then $G$ can be written as $X y^{-1}$, where $X \in \mathcal{T}_{0}^{n \times m}$, 
$y \in 9$. We will now show that there exists a matrix $U \in$ $\mathcal{H}_{0}^{(n+m) \times(n+m)}$ with $\operatorname{det} U=1$, such that

$$
U\left[\begin{array}{c}
y I_{m} \\
X
\end{array}\right]=\left[\begin{array}{l}
T \\
0_{n \times m}
\end{array}\right] .
$$

By Lemma 2.2 this is enough to show that $G$ has an ref.

We will show that $U$ can be constructed as a product of $n m$ unimodular matrices $U_{11}, \cdots, U_{n m}$, and explicitly demonstrate $U_{11}$. The rest of the matrices $U_{12}, \cdots, U_{n m}$ can be constructed in an entirely analogous manner.

By Assumption 3), $y$ and $x_{11}$ generate a principal ideal. Let $r \in \mathcal{K}_{0}$ be a generator of this ideal. Then there exist $p, q, a, b$ in $\mathcal{K}_{0}$ such that

$$
\begin{gathered}
p y+q x_{11}=r \\
y=a r, \quad x_{11}=b r .
\end{gathered}
$$

By Assumption 2), $r \in \mathscr{G}$. Now note that

$$
\begin{aligned}
a x_{11} & =b y \\
p a+q b & =1
\end{aligned}
$$

and define
The proof that $G$ also has an lcf is entirely analogous and is left to the reader.

As a corollary to Theorem 2.1 we have the following result, which is stated in [12] in a somewhat different form.

Corollary 2.3: Suppose $\mathcal{H}$ is a Bezout domain. Then all finitely generated left ideals and right ideals in $\mathcal{C}^{n \times n}$ are principal.

\section{Further Examples}

In this section we apply Theorem 2.1 to the rings of Examples 2.1 and 2.2. It appears that Theorem 2.1 is primarily useful in studying distributed (one-dimensional) systems; it does not appear to be very useful in studying multidimensional systems.

Example 2.3: Let $\mathscr{K}=l_{1}$, as in Example 2.1. To identify a subset $\mathcal{G}$ of the quotient field of $\mathcal{H}$ such that every fraction in $\mathcal{G}$ has a coprime factorization, we define

$$
\begin{aligned}
\mathscr{H}_{0} & =\{\tilde{f} \in H: z \mapsto \tilde{f}(r z) \in \mathcal{K} \text { for some } r>1\} \\
\Phi & =\{0\} .
\end{aligned}
$$

In effect, $\mathcal{K}_{0}$ consists of functions of the form

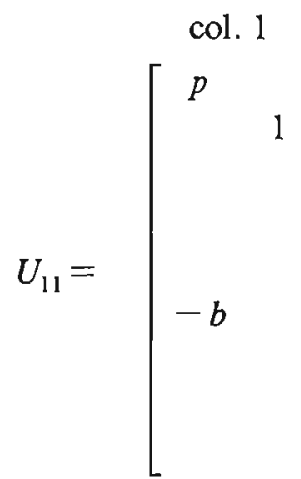

col. $(m+1)$

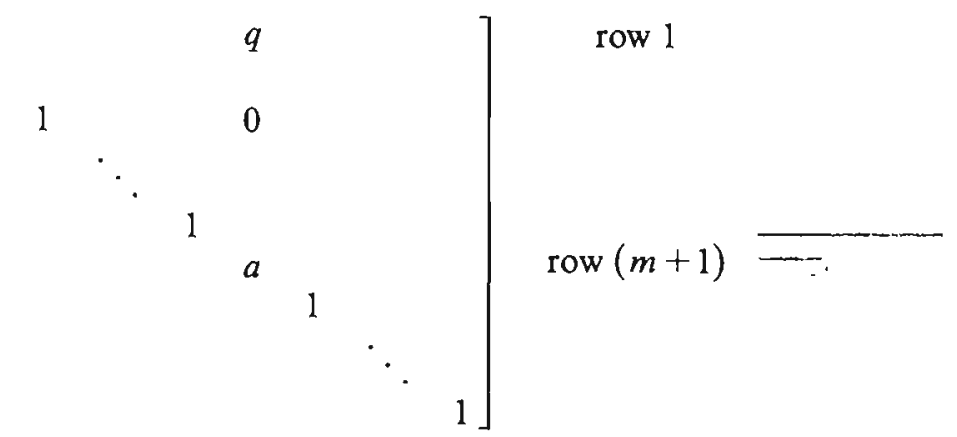

Now $\operatorname{det} U_{11}=1$ [because of (2.30)], and

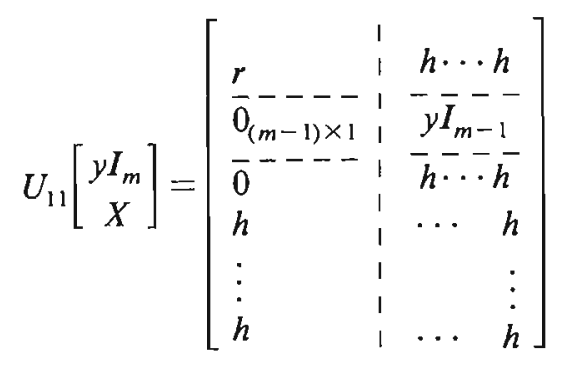$$
\tilde{f}(z)=\sum_{i=0}^{\infty} f_{i} z^{i}
$$

where the sequence $\left\{r^{i} f_{i}\right\}$ is absolutely summable for some $r>1$. Every function in $\mathcal{H}$ is analytic over the open unit disk; further, every function in $\mathcal{K}_{0}$ is analytic over the disk $|z|<r$ for some $r>1$, and is hence analytic over the closed unit disk. As a result, if $\tilde{f} \in \mathscr{K}_{0}$ and $\tilde{g} \in \mathcal{K}_{0} \backslash 0$, both functions have only a finite number of isolated zeros of finite degree inside the closed unit disk. Hence, the gcd of $\tilde{f}$ and $\tilde{g}$ inside the closed unit disk is some polynomial $\phi(z)$, which belongs to $\mathcal{K}_{0}$ (since all polynomials belong to $\mathcal{K}_{0}$ ). So if 'we define

whe we $h$ as a generic symbol to indicate element of $\mathscr{H}_{0}$. Thus, as a result of premultiplication by $U_{11}$, a zero has been created in the $(m+1,1)$ position. Since $r \in \mathcal{G}$, the procedure can be continued to create zeros in the first column in rows $m+1$ to $m+m$. The same procedure can then be applied to all subsequent columns. In this way one can construct a matrix $U$ such that (2.26) is true (and, in addition, $T$ is upper triangular). It now follows from Lemma 2.2 that $G$ has an rcf.

$$
\tilde{f}_{1}(z)=\tilde{f}(z) / \phi(z), \quad g_{1}(z)=\tilde{g}(z) / \phi(z)
$$

then $\tilde{f}_{1}, \tilde{g}_{1} \in \mathcal{H}_{0}$ have no common zeros in the closed unit disk and are coprime. Hence, $\phi$ is the gcd of $\tilde{f}$ and $\tilde{g}$ in $\mathcal{H}$, and the ideal generated by $f$ and $g$ coincides with the ideal 
generated by $\phi$. In conclusion, every element $\tilde{f} / \tilde{g}$, where $\tilde{f} \in \mathcal{K}_{0}$ and $\tilde{g} \in \mathcal{K}_{0} \backslash 0$, has a coprime factorization in $\mathcal{K}$.

In this example, we chose $\mathscr{G}=\mathcal{K}_{0} \backslash 0$ to achieve the greatest possible generality. However, from the point of view of well-posedness, it may be more natural to take

$$
g=\left\{\tilde{f} \in \mathcal{K}_{0}: \tilde{f}(0) \neq 0\right\} .
$$

Example 2.4: Let $\mathcal{K}=\mathcal{Q}$, as in Example 2.2. In order to identify a subset of the quotient field of $Q$ where every element has a coprime factorization, we must look for a subring $\mathcal{K}_{0}$ of $Q$ and a subset $\mathcal{G}$ of $\mathcal{K}_{0}$ satisfying Assumptions 1)-3) of Theorem 2.1. This is done in [6], although the point of view is not the same as it is here.

To relate the present approach to that of [6], we first define the set $Q$, as follows:

$\mathbb{Q}_{-}=\left\{f(\cdot)\right.$ : the distribution $t \mapsto e^{\sigma t} f(t) \in \mathbb{Q}$ for some $\left.\sigma>0\right\}$.

The set $\mathcal{Q}_{-}$is a subring of $\mathbb{Q}$, although $(\mathbb{Q},\|\cdot\| \mathscr{Q})$ is not a Banach space. We take $\mathcal{H}_{0}=\mathscr{Q}_{-}$. Next we define

$g=\left\{f(\cdot) \in \mathbb{Q}_{-}:\right.$there exists an $r>0$ such that

$$
\left.\inf _{s \in C_{+},|s| \geqslant r}|\hat{f}(s)|>0\right\} .
$$

In effect, 9 consists of those distributions in $\mathbb{Q}$ whose Laplace transforms are "bounded away from zero at infinity." Clearly, 9 satisfies Assumptions 1) and 2) of Theorem 2.1. To verify the principal ideal property we begin by observing that whenever $f \in Q_{-}$, its Laplace transform $\hat{f}$ is analytic over some open half-plane $\operatorname{Re} s>-\sigma$, and hence over the closed right half-plane $C_{+}$. Hence, all zeros of $\hat{f}$ in $C_{+}$are isolated and of finite order. Now, suppose that $f$ actually belongs to 9 . Then all zeros of $f$ in $C_{+}$are in some compact subset of $C_{+}$, in view of (2.39). This means that, in fact, $\hat{f}$ has only a finite number of zeros, each of finite order, in $C_{+}$. Now let $f \in \mathbb{Q}, g \in \mathscr{G}$; in view of the preceding discussion $\hat{f}$ and $\hat{g}$ have only a finite number of common zeros, each of finite order, in $C_{+}$. Let these be $s_{1}, \cdots, s_{n}$ with multiplicities $m_{1}, \cdots, m_{n}$, and let $\phi(s)$ be the polynomial

$$
\phi(s)=\prod_{i=1}^{n}\left(s-s_{i}\right)^{m_{i}} .
$$

We now have the following.

Proposition 2.1: Define

$$
\hat{f}_{1}(s)=\hat{f}(s) \cdot \frac{(s+1)^{n}}{\phi(s)}, \quad \hat{g}_{1}(s)=\hat{g}(s) \cdot \frac{(s+1)^{n}}{\phi(s)} .
$$

Then $f_{1}, g_{1} \in \mathbb{Q}$ _ and are coprime. Moreover, the ideal generated by $f$ and $g$ is the same as the principal ideal generated by $h$, where $\hat{h}(s)=\phi(s) /(s+1)^{n}$.
Proof: To make the idea clear, consider the simple case where $\phi(s)=\left(s-s_{0}\right)^{n}$, where $s_{0} \in C_{+}$. Thus, both $\hat{f}$ and $\hat{g}$ have zeros of multiplicity at least $n$ at $s_{0}$. First, we claim that

$$
\hat{f}(s) \cdot \frac{s+1}{s-s_{0}} \in \hat{Q}
$$

where $\hat{\mathcal{E}}$ _ denotes the set of Laplace transforms of the elements of $Q_{-}$. To prove (2.42) observe that

$$
\hat{f}(s) \cdot \frac{s+1}{s-s_{0}}=\hat{f}(s)+\frac{\left(1+s_{0}\right)}{s-s_{0}} \hat{f}(s) .
$$

Note that $\left(1+s_{0}\right)$ is a number. Now by [6, Theorem 2.2],

$$
\hat{f}(s) \cdot \frac{\left(1+s_{0}\right)}{s-s_{0}}=\frac{f\left(s_{0}\right)}{s-s_{0}}+\hat{f}_{\rho}(s)=\hat{f}_{\rho}(s)
$$

where $f_{\rho} \in Q_{-}$. Observe also that $\hat{f}_{\rho}$ has a zero of order $n-1$ at $s_{0}$. Repeating the argument shows that $\hat{f}_{1}(s)=(s+$ $1)^{n} f(s) /\left(s-s_{0}\right)^{n} \in Q_{-}$. The proof in the more general case where $\phi(s)$ is a product of several factors of the form $\left(s-s_{i}\right)^{n_{i}}$ is now routine. Thus, we have established that $f_{1}, g_{1} \in \mathbb{Q}_{-}$.

Next, we show that $f_{1}, g_{1}$ are coprime. Clearly, $g_{1} \in 9$ because $g \in 9$. Hence, by [6, Corollary 2.1], $f_{1}$ and $g_{1}$ are coprime provided $\hat{f}_{1}, \hat{g}_{1}$ have no common zeros in $C_{+}$. From the definition of $\phi(s)$ and from (2.41), it follows that $\hat{f}_{1}, \hat{g}_{1}$ have no common zeros in $C_{+}$, and that $f_{1}, g_{1}$ are therefore coprime.

Finally, we see from (2.41) that

$$
\hat{f}(s)=\hat{f}_{1}(s) \hat{h}(s), \quad \hat{g}(s)=\hat{g}_{1}(s) \hat{h}(s) .
$$

Since $f_{1}$ and $g_{1}$ are coprime, $h$ is a gcd in $Q$ of $f$ and $g$; therefore, the ideal generated by $f$ and $g$ is the same as the ideal generated by $h$.

The preceding discussion shows that every element of $\mathcal{G}=\mathscr{Q}^{-1} \mathbb{Q}$ has a coprime factorization in $Q$. This is exactly the algebra introduced in [6]. Moreover, in view of Theorem 2.1 every element of $\mathcal{G}^{n \times m}$ has both an rcf and an lcf. This was proved in [7] using slightly different methods.

\section{Stabilization}

In this section we study the existence and characterization of the set of all compensators that stabilize a given plant. We also extend the concept of a "proper" plant and a "strictly proper" plant to an abstract setting and prove two very important and general results: 1) every strictly proper plant can be stabilized by a proper compensator; 2) all compensators that stabilize a strictly proper plant must be proper. Finally, we show that under slightly stronger assumptions almost all plants with an equal number of inputs and outputs can be stabilized by an appropriate compensator. We begin by reviewing feedback stability.

Note that, throughout the rest of the paper, we use $e_{r}^{n \times m}$ (resp. $e_{l}^{n \times m}$ ) to denote the set of all $G \in \mathscr{F}^{n \times m}$ that have an 


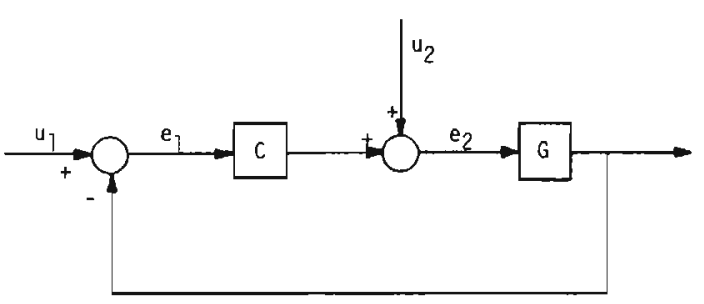

Fig. 1. Closed-loop system.

rcf (resp. an lcf). Also, we use $e^{n \times m}$ to denote $e_{r}^{n \times m} \cap e_{1}^{n \times m}$; i.e., $e^{n \times m}$ consists of all $G \in F^{n \times m}$ that have both an rcf and an lcf.

\section{A. Closed-Loop Stability Revisited}

In this subsection we review the problem of the closedloop stability of the system shown in Fig. 1. Suppose $C \in \mathscr{F}^{m \times n}, G \in \mathscr{F}^{n \times m}$, and that $\operatorname{det}\left(I_{m}+C G\right)=\operatorname{det}\left(I_{n}+\right.$ $G C) \neq 0$. Then the relation between $u_{1}, u_{2}, e_{1}, e_{2}$ can be expressed as

$$
\left[\begin{array}{l}
e_{1} \\
e_{2}
\end{array}\right]=\left[\begin{array}{cc}
\left(I_{n}+G C\right)^{-1} & -G\left(I_{m}+C G\right)^{-1} \\
C\left(I_{n}+G C\right)^{-1} & \left(I_{m}+C G\right)^{-1}
\end{array}\right]\left[\begin{array}{l}
u_{1} \\
u_{2}
\end{array}\right] .
$$

In (3.1) both $\left(I_{n}+G C\right)^{-1}$ and $\left(I_{m}+C G\right)^{-1}$ occur, which is not very convenient. To avoid this we can either rewrite everything in terms of $\left(I_{n}+G C\right)^{-1}$ or in terms of $\left(I_{m}+\right.$ $C G)^{-1}$ using standard matrix identities. This gives

$$
\left[\begin{array}{l}
e_{1} \\
e_{2}
\end{array}\right]=\left[\begin{array}{cc}
\left(I_{n}+G C\right)^{-1} & -\left(I_{n}+G C\right)^{-1} G \\
C\left(I_{n}+G C\right)^{-1} & I_{m}-C\left(I_{n}+G C\right)^{-1} G
\end{array}\right]\left[\begin{array}{l}
u_{1} \\
u_{2}
\end{array}\right]
$$

if we express everything in terms of $\left(I_{n}+G C\right)^{-1}$, and

$$
\left[\begin{array}{l}
e_{1} \\
e_{2}
\end{array}\right]=\left[\begin{array}{cc}
I_{n}-G\left(I_{m}+C G\right)^{-1} C & -G\left(I_{m}+C G\right)^{-1} \\
\left(I_{m}+C G\right)^{-1} C & \left(I_{m}+C G\right)^{-1}
\end{array}\right]\left[\begin{array}{l}
u_{1} \\
u_{2}
\end{array}\right]
$$

if we express everything in terms of $\left(I_{m}+C G\right)^{-1}$. Let us label the $(n+m) \times(n+m)$ matrix in (3.2) and (3.3) as $H$, and label its four submatrices as $H_{11}, H_{12}, H_{21}, H_{22}$, respectively. We say that the pair $(G, C)$ is stable if $\operatorname{det}\left(I_{n}\right.$ $+G C)=\operatorname{det}\left(I_{m}+C G\right) \neq 0$, and $H \in \mathcal{F}^{(n+m) \times(n+m)}$. We also say that $C$ stabilizes $G$ if $(G, C)$ is stable.

Lemma 3.1: Suppose $G \in \mathcal{C}_{r}^{n \times m}, C \in \mathcal{C}_{1}^{m \times n}$, and let $\left(N_{p}, D_{p}\right)$ be any rcf of $G,\left(\tilde{D}_{c}, \bar{N}_{c}\right)$ any lcf of $C$. Under these conditions the pair $(G, C)$ is stable if and only if

$$
\Delta_{1}=\tilde{D}_{c} D_{p}+\tilde{N}_{c} N_{p}
$$

is unimodular in $\mathcal{H}^{\mathrm{m} \times m}$ ?

\footnotetext{
${ }^{9}$ Note that we do not have here the technical assumptions that $\left(N_{p}, \bar{D}_{c} D_{p}\right)$ are right-coprime and that $\left(\tilde{D}_{c} D_{p}, \tilde{N}_{c}\right)$ are left-coprime, as in $[8]$.
}

Proof: "If": Suppose $\Delta_{1}$ is unimodular, so that $\Delta_{1}^{-1} \in$ $\mathcal{H}^{m \times m}$. First, since $\operatorname{det}\left(I_{m}+G C\right)=\operatorname{det}\left[\tilde{D}_{c}^{-1} \Delta_{1} D_{p}^{-1}\right]$, we see that $\operatorname{det}\left(I_{m}+G C\right) \neq 0$. Next it is easy to verify by direct substitution in (3.3) that

$$
H=\left[\begin{array}{cc}
I_{n}-N_{p} \Delta_{1}^{-1} \tilde{N}_{c} & -N_{p} \Delta_{1}^{-1} \tilde{D}_{c} \\
D_{p} \Delta_{1}^{-1} \tilde{N}_{c} & D_{p} \Delta_{1}^{-1} \tilde{D}_{c}
\end{array}\right]
$$

Since $\Delta_{1}^{-1} \in \mathcal{K}^{m \times m}$, it is immediate that $H \in \mathcal{H}^{(n+m) \times(n+m)}$.

"Only if": Suppose $(G, C)$ is stable. ${ }^{10}$ Then by definition $\operatorname{det}\left(I_{m}+C G\right) \neq 0$ and $H \in \mathcal{T}^{(n+m) \times(n+m)}$. Moreover, if $\Delta_{1}$ is defined by (3.4), then $\operatorname{det} \Delta_{1}=\operatorname{det}\left[\tilde{D}_{c}\left(I_{m}+C G\right) D_{p}\right] \neq 0$, and $\Delta_{1}^{-1}$ is well defined in $\mathscr{F}^{m \times m}$, where $\mathscr{F}$ is the quotient field of $\mathcal{H}$. Also, $H$ is given by (3.5). Now since $H \in$ $\mathcal{H}^{(n+m) \times(n+m)}$ and $I_{n} \in \mathcal{H}^{n \times n}$, it follows from (3.5) that

$$
\left[\begin{array}{ll}
N_{p} \Delta_{1}^{-1} \tilde{N}_{c} & N_{p} \Delta_{1}^{-1} \tilde{D}_{c} \\
D_{p} \Delta_{1}^{-1} \tilde{N}_{c} & D_{p} \Delta_{1}^{-1} \tilde{D}_{c}
\end{array}\right] \in \mathcal{K}^{(n+m) \times(n+m)} .
$$

Since $N_{p}, D_{p}$ are right-coprime and $\tilde{N}_{c}, \tilde{D}_{c}$ are left-coprime, there exist matrices $P \in \mathcal{K}^{m \times n}, \tilde{P}_{c} \in \mathcal{K}^{n \times m}, Q, \tilde{Q}_{c} \in \mathcal{K}^{m \times m}$ such that

$$
P N_{p}+Q D_{p}=I_{m}, \quad \tilde{N}_{c} \tilde{P}_{c}+\tilde{D}_{c} \tilde{Q}_{c}=I_{m} .
$$

Thus

$$
\Delta^{-1}=\left[\begin{array}{ll}
P & Q
\end{array}\right]\left[\begin{array}{ll}
N_{p} \Delta_{1}^{-1} \tilde{N}_{c} & N_{p} \Delta_{1}^{-1} \tilde{D}_{c} \\
D_{p} \Delta_{1}^{-1} \tilde{N}_{c} & D_{p} \Delta_{1}^{-1} \tilde{D}_{c}
\end{array}\right]\left[\begin{array}{c}
\tilde{P}_{c} \\
\tilde{Q}_{c}
\end{array}\right] \in \mathcal{K}^{m \times m}
$$

which shows that $\Delta_{1}$ is unimodular.

The analog of Lemma 3.1 for the case $G \in \mathcal{C}_{l}^{n \times m}, C \in$ $e_{r}^{m \times n}$ is obvious and need not be stated explicitly.

Once we have the general characterization of feedback stability as given in Lemma 3.1 and its analog alluded to above, we can readily characterize all compensators $C$ that stabilize a given plant $G$. The proof of Lemma 3.2 below is omitted, as it closely follows that of $[8$, Theorem 3$] .{ }^{11}$

Lemma 3.2: Suppose $G \in \mathrm{e}^{n \times m}$. Let $\left(N_{p}, D_{p}\right),\left(\tilde{D}_{p}, \tilde{N}_{p}\right)$ be any rcf and lcf of $G$, and select matrices $P, \tilde{P}, Q, \tilde{Q}$ such that

$$
P N_{p}+Q D_{p}=I_{m}, \quad \tilde{N}_{p} \dot{\tilde{P}}+\tilde{D}_{p} \tilde{Q}=I_{n} .
$$

Then $C \in \mathbb{C}_{I}^{m \times n}$ stabilizes $G$ if and only if $C$ has an lcf $\left(\tilde{D}_{c}, \tilde{N}_{c}\right)$ with

$$
\tilde{D}_{c}=Q-R \tilde{N}_{p}, \tilde{N}_{c}=P+R \tilde{D}_{p} \quad \text { for some } R \in \mathcal{K}^{m \times n} \text {. }
$$

$C \in \mathcal{C}_{r}^{m \times n}$ stabilizes $G$ if and only if $C$ has an $\operatorname{rcf}\left(N_{c}, D_{c}\right)$ with

$$
D_{c}=\tilde{Q}-N_{p} S, \quad N_{c}=\tilde{P}+D_{p} S \quad \text { for some } S \in \mathcal{H}^{m \times n} .
$$

\footnotetext{
${ }^{10}$ We are grateful to C. Desoer for supplying this simple proof.

"In [8], the authors only give the formula (3.9), but (3.10) is easily derivable using the same methods.
} 


\section{B. Existence and Properness of Stabilizing Compensators}

Suppose we are given a plant $G \in e^{n \times m}$. Select an rcf $\left(N_{p}, D_{p}\right)$ and an $\operatorname{lcf}\left(\tilde{D}_{p}, \tilde{N}_{p}\right)$ of $G$, and matrices $P, \tilde{P}, Q, \tilde{Q}$ such that (3.8) holds. Then the set of all $C \in \mathcal{C}_{l}^{m \times n}$ that stabilize $G$ is given by

$$
\begin{aligned}
& S_{l}(G)=\left\{\left(Q-R \tilde{N}_{p}\right)^{-1}\left(P+R \tilde{D}_{p}\right):\right. \\
& \left.\quad R \in \mathcal{K}^{m \times n} \text { and } \operatorname{det}\left(Q-R \tilde{N}_{p}\right) \neq 0\right\} .
\end{aligned}
$$

In other words, we let $R$ vary over all elements of $\mathcal{K}^{m \times n}$ such that $\operatorname{det}\left(Q-R \tilde{N}_{p}\right) \neq 0$. The set of all corresponding ratios $\left(Q-R \tilde{N}_{p}\right)^{-1}\left(P+R \tilde{D}_{p}\right)$ is precisely the set of all $C$ in $\bigcup_{l}^{m \times n}$ that stabilize $G$. The analogous result for $C \in \bigcup_{r}^{m \times n}$ is as follows: the set of all $C \in e^{m \times n}$ that stabilize $G$ is given by

$$
\begin{aligned}
& \mathcal{S}_{r}(G)=\left\{\left(\tilde{P}+D_{p} S\right)\left(\tilde{Q}-N_{p} S\right)^{-1}:\right. \\
& \left.\quad S \in \mathcal{K}^{m \times n} \text { and } \operatorname{det}\left(\tilde{Q}-N_{p} S\right) \neq 0\right\} .
\end{aligned}
$$

From the point of view of compensator design, two types of questions arise. 1) given a plant $G$, what is the set of $R \in \mathcal{K}^{m \times n}$ such that $\operatorname{det}\left(Q-R \tilde{N}_{p}\right) \neq 0$ ? Is it a nonempty set; i.e., can $G$ be stabilized by some compensator? 2) for what class of plants $G$ can we find stabilizing compensators that are "proper"? (And what is meant by a "proper" compensator in a general ring theory setting?) We shall answer each type of question in turn. First, we show that in the scalar case every $g \in \mathcal{C}$ can be stabilized by a $c \in \mathcal{C}$. We also show that if $\mathcal{K}$ is a normed algebra, then the set of $g \in e^{n \times m}$ for which $\delta_{r}(g)$ is nonempty (i.e., the set of $g \in e^{n \times m}$ that can be stabilized) is an open, dense subset of $e^{n \times n}$. These results provide answers to the first set of questions. Next, we define the concepts of "proper" and "strictly proper" plants in the abstract ring-theoretic setting, and prove two results: 1) every strictly proper plant can be stabilized by a proper compensator, and 2) all compensators that stabilize a strictly proper plant are proper.

Proposition 3.I: Corresponding to every $g \in \mathcal{C}$ there exists a $c \in \mathcal{C}$ that stabilizes $g$.

Proof: In the scalar case there is no distinction between lcf's and rcf's. Thus, given $g \in \mathcal{C}$, let $\left(n_{p}, d_{p}\right)$ be any coprime factorization of $g$, and select $p, q$ in $\mathcal{K}$ such that

$$
p n_{p}+q d_{p}=1 \text {. }
$$

It is clear from (3.13) that $n_{p}$ and $q$ cannot both be zero. Now if $q=0$, let $r=1$, while if $q \neq 0$, let $r=0$. In this way it is always possible to select $r \in \mathcal{K}$ such that $q-r n_{p} \neq 0$. For this choice of $r$, the compensator $c=\left(p+r d_{p}\right) /(q-$ $\left.r n_{p}\right)$ is well defined and stabilizes $g$.

Proposition 3.2: Consider the case $m=n$, and suppose $\mathcal{K}$ is a normed algebra. Suppose $G \in e^{n \times m}$ and that $\operatorname{det} G$ $\neq 0$. Then there exists $C \in \mathcal{C}_{1}^{n \times m}$ (resp. a $\bar{C} \in \mathcal{C}_{r}^{n \times m}$ ) that stabilizes $G$.

Proof: Let $\left(\tilde{D}_{p}, \tilde{N}_{p}\right),\left(N_{p}, D_{p}\right)$ be any rcf and lcf of $G$, and select $P, Q, \tilde{P}, \tilde{Q}$ to satisfy (3.8). Since $\operatorname{det} G \neq 0$, it follows that $\operatorname{det} N_{p} \neq 0$, $\operatorname{det} \tilde{N}_{p} \neq 0$. Now consider the quantity $Q-R \tilde{N}_{p}$ with $R=\lambda I_{n}$, where $\lambda$ is a real number. Since

$$
\operatorname{det}\left(Q-\lambda \tilde{N}_{p}\right)=\lambda^{n} \operatorname{det}\left(\lambda^{-1} Q-\tilde{N}_{p}\right)
$$

and since $\operatorname{det} \tilde{N}_{p} \neq 0$, we have that $\operatorname{det}\left(Q-\lambda \tilde{N}_{p}\right) \neq 0$ for sufficiently large $\lambda$. Hence, for sufficiently large $\lambda, C=(Q$ $\left.-\lambda \tilde{N}_{p}\right)^{-1}\left(P+\lambda \tilde{D}_{0}\right)$ is well defined, belongs to $e_{l}^{n \times n}$, and stabilizes $G$. The existence of a stabilizing $\bar{C} \in \mathcal{C}_{r}^{n \times n}$ is proved in the same way.

Although we have not yet talked about topology, the next result logically belongs here, so we state it now. The proof is immediate from Lemma 4.2.

Proposition 3.3: Suppose $\mathcal{K}$ is a normed algebra, and consider the set $C^{n \times n}$ with the topology of Definition 4.1. Then the set of $G \in e^{n \times n}$ such that $\operatorname{det} G \neq 0$. (in which case $G$ can be stabilized) is open and dense in $e^{n \times n}$.

Now we come to a study of "proper" compensators for "strictly proper" plants. In the case of rational transfer functions, we say that $\hat{g}(s)$ is proper (strictly proper) if the degree of the denominator polynomial of $\hat{g}(s)$ is greater than or equal to (greater than) the degree of the numerator polynomial of $\hat{g}(s)$. Equivalently, $\hat{g}(s)$ is proper (strictly proper) if $\hat{g}(\infty)$ is finite (zero). This " $s$ plane" definition tends to obscure the fact that one can define the concepts of a proper and strictly proper plant in an abstract ringtheoretic setting.

Definition 3.I: Given the ring $\mathcal{K}$, let $\mathscr{K}$ be an ideal ${ }^{12}$ in $\mathcal{K}, \mathscr{K} \neq \mathcal{K}$. We define

$$
\mathscr{P}=\{g \in \mathscr{F}: g=n / d \text { for some } n \in \mathcal{K}, d \in \mathcal{K} \backslash \mathcal{K}\} \text {. }
$$

$$
\varsigma \mathscr{P}=\{g \in \mathscr{F}: g=n / d \text { for some } n \in \mathscr{K}, d \in \mathcal{K} \backslash \mathscr{K}\} \text {. }
$$

We shall say that $g \in \mathscr{F}$ is proper if $g \in \mathscr{Q}$, strictly proper if $g \in \mathcal{S} \mathscr{P}$.

We digress briefly to show that the above definitions are consistent with conventional usage. Suppose we wish to study the case where $\mathscr{F}$ is the set of all rational functions. Then $\mathscr{F}$ is the quotient field associated with the ring $\mathcal{K}$ of proper stable rational functions. ${ }^{3}$ (That is, $h(s)=$ $p(s) / q(s)$ belongs to $\mathcal{K}$ if and only if $\operatorname{deg} p \leqslant \operatorname{deg} q$, and all zeros of $q$ have negative real parts.) Now let $\mathscr{K}$ denote the set of all strictly proper elements in $\mathcal{K}$ (i.e., $\mathcal{K}$ is the set of strictly proper stable functions). Then $\mathscr{K}$ is an ideal in $\mathcal{K}$, and $\mathcal{K}$ is not all of $\mathcal{K}$. Also, $\hat{f}(s) \in \mathcal{K} \backslash \mathcal{K}$ if and only if $\hat{f}(\infty) \neq 0$; i.e., the relative degree of the rational function $\hat{f}$ is zero. Now, given an arbitrary rational function $\hat{r}(s)=$ $p(s) / q(s)$, let $\alpha=\operatorname{deg} p, \beta=\operatorname{deg} q, \gamma=\max \{\alpha, \beta\}$, and write

\footnotetext{
${ }^{12}$ Recall that $\mathscr{X}$ is an ideal in $\mathcal{X}$ if $\mathcal{K}$ is a subring of $\mathcal{K}$, and if $x y \in \mathscr{K}$ whenever $x \in \mathcal{K}$ and $y \in \mathcal{K}$ K is a proper ideal in $\mathcal{K}$, since $\mathcal{K}$ is not all of $\mathcal{K}$. However, to avoid confusion we shall simply call $\mathcal{K}$ an ideal in $\mathcal{K}$, it being understood that $\mathcal{K}$ is not all of $\mathcal{K}$. The ideal $\mathcal{K}$ is prime if $x \in \mathcal{K}$, $y \in \mathcal{K}$, and $x y \in \mathcal{K}$ implies that either $x \in \mathcal{K}$ or $y \in \mathcal{K}$.

${ }^{13}$ In this paragraph quantities with " $"$ denote rational functions, and those without denote polynomials.
} 


$$
\hat{r}(s)=\frac{p(s) /(s+1)^{\gamma}}{q(s) /(s+1)^{\gamma}}
$$

Then $\hat{r}(s) \in \mathscr{P}$ (resp. $\mathcal{S} \mathscr{P}$ ) if $\alpha \leqslant \beta$ (resp. $\alpha<\beta$ ). Conversely, suppose $\hat{r}(s)=\hat{n}(s) / \hat{d}(s) \in \mathscr{P}$ (resp. $\delta \mathscr{P}$ ). Then $\hat{r}(\infty)=$ $\hat{n}(\infty) / \hat{d}(\infty)$ is finite so that $\hat{r}(s)$ is proper in the usual sense (resp. $\hat{r}(\infty)=0$, so that $\hat{r}(s)$ is strictly proper in the usual sense).

Lemma 3.3: Suppose $\mathcal{K}$ is a prime ideal in $\mathcal{K}$. Then both $\mathscr{P}$ and $\delta \mathscr{P}$ are subrings of $\mathscr{F}$.

Proof: If $\mathcal{K}$ is a prime ideal, then $\mathcal{K} \backslash \mathcal{K}$ is closed under multiplication.

In what follows it is assumed that $\mathcal{K}$ is an ideal in $\mathcal{K}$ and that $\mathcal{K}$ is not all of $\mathcal{K}$. A few results require $\mathcal{K}$ to be a prime ideal, in which case this is stated explicitly.

We now state a lemma that is used repeatedly in the sequel.

Lemma 3.4: Suppose $Q \in \mathcal{H}^{n \times n}$, det $Q \in \mathcal{H} \backslash \mathcal{K}$ and $M \in$ $\mathscr{K}^{n \times n}$. Then $\operatorname{det}(Q+M) \in \mathcal{K} \backslash \mathcal{K}$.

Proof: By definition, we have

$$
\operatorname{det}(Q+M)=\sum_{\phi} \operatorname{sign}(\phi) \prod_{i=1}^{n}(Q+M)_{i, \phi(i)}
$$

where $\phi$ denotes a permutation of $\{1, \cdots, n\}$ and the summation is over all permutations. Now, note that

$$
\prod_{i=1}^{n}(Q+M)_{i, \phi(i)}=\left[\prod_{i=1}^{n} Q_{i, \phi(i)}\right]+r_{\phi}
$$

where $r_{\phi}$ is some element of $\mathcal{K}^{14}$ Hence

$$
\begin{aligned}
\operatorname{det}(Q+M) & =\sum_{\phi} \operatorname{sign}(\phi)\left[\prod_{i=1}^{n} Q_{i, \phi(i)}+r_{\phi}\right] \\
& =\operatorname{det} Q+r, \quad \text { where } r \in \mathcal{K} .
\end{aligned}
$$

Since $\operatorname{det} Q \in \mathcal{H} \backslash \mathcal{K}$ and $\mathscr{K}$ is a subring, we see that $\operatorname{det}(Q$ $+M) \in \mathcal{K} \backslash \mathscr{K}$.

Definition 4.2: An rcf $(N, D)$ of an element $G \in \mathcal{C}_{r}^{n \times m}$ is said to be strictly proper if $N \in \mathcal{K}^{n \times m}$.

Lemma 3.5: Suppose $(N, D)$ is a strictly proper rcf of $G \in \mathcal{C}_{r}^{n \times m}$. Then det $D \in \mathcal{H} \backslash \mathscr{K}$.

Proof: Since $(N, D)$ is right-coprime, there exist $P, Q$ such that

$$
P N+Q D=I_{m} .
$$

Hence, $Q D=I_{m}-P N$ and $\operatorname{det}\left(I_{m}-P N\right)=\operatorname{det} Q \cdot \operatorname{det} D$. Now, det $I_{m}=1 \in \mathcal{K} \backslash \mathcal{K}$, and $P N \in \mathscr{K}^{m \times n}$ since $N \in \mathscr{K}^{n \times m}$. Therefore, by Lemma 3.4 it follows that $\operatorname{det}\left(I_{m}-P N\right) \in \mathcal{K}$ $\checkmark \mathcal{K}$. Hence, det $Q \in \mathcal{K} \backslash \mathcal{K}$ and det $D \in \mathcal{K} \backslash \mathcal{K}$, since both are factors of $\operatorname{det}\left(I_{m}-P N\right)$.

Lemma 3.6: Suppose $G \in \mathcal{C}_{r}^{n \times m}$ has a strictly proper rcf $(N, D)$. Then, $G \in \mathcal{S} \mathscr{\rho} n \times m$.

\footnotetext{
${ }^{14}$ The reasoning is as follows. Consider a product $\prod_{i=1}^{n}\left(a_{i}+b_{i}\right)$ where $b_{i} \in \mathcal{K}$ for all $i$. Then $\prod_{i=1}^{n}\left(a_{i}+b_{i}\right)=\prod_{i=1}^{n} a_{i}+\sum$ products involving at least one of the $b_{i}{ }^{\prime} \mathrm{s}=\prod_{i=1}^{{ }^{i}=1} a_{i}+$ an element of $\mathfrak{K}^{\prime}$.
}

Proof: We have $G=N D^{-1}=N \cdot \operatorname{Adj} D \cdot(\operatorname{det} D)^{-1}$ Since det $D \in \mathcal{H} 、 \mathcal{K}$ by Lemma 3.5 and $N \cdot$ Adj $D \in \mathcal{K}^{n \times m}$, the conclusion follows.

The lcf analogs of Definition 4.2 and Lemmas 3.5 and 3.6 are obvious and need not be stated explicitly.

Lemma 3.6 and its lcf analog show that if $G$ has a strictly proper rcf or lcf, then every element of $G$ must be strictly proper. The converse may not always be true; i.e., even if $G \in \delta \rho^{n \times m}$, it may not have a strictly proper rcf or lcf. However, in the important special case where $\mathcal{H}$ is the set of proper stable rational functions and $\mathcal{F}$ is the set of rational functions, every $G \in \delta \mathcal{P}^{n \times m}$ has both a strictly proper lcf and a strictly proper rcf. This is a consequence of the next result.

Lemma 3.7: Suppose $\mathcal{K}$ is a Bezout domain, and let $\mathscr{K}$ be a proper prime ideal in $\mathcal{H}$. Then every $G \in \delta \rho^{n \times m}$ has both a strictly proper lcf and a strictly proper ref.

The proof is long but straightforward, based on Lemma $2.2 \mathrm{It}$ is omitted here in the interests of brevity and can be found in [28].

We now come to the important result in this regard.

Theorem 4.1: Suppose $G \in \mathcal{C}^{m \times n}$ has both a strictly proper rcf and a strictly proper lcf. Then there exists a proper compensator $C$ that stabilizes $G$. Moreover, every compensator $C \in \mathcal{C}_{r}^{n \times m} \cup \mathcal{C}_{l}^{n \times m}$ that stabilizes $G$ must be proper.

Proof: Let $\left(N_{p}, D_{p}\right),\left(\tilde{D}_{p}, \tilde{N}_{p}\right)$ be a strictly proper rcf and lcf of $G$, and select $P, Q, \tilde{P}, \tilde{Q}$ such that (3.8) is satisfied. Then, as shown in the proof of Lemma 4.2 we have $\operatorname{det} Q \in \mathcal{H} \backslash \mathcal{K}$. Next, by Lemma 3.4 it follows that $\operatorname{det}\left(Q-R \tilde{N}_{p}\right) \in \mathcal{H} \backslash \mathcal{K}$ for all $R \in \mathcal{H}^{m \times n}$. In particular, since $0 \in \mathcal{K}$, we see that $\operatorname{det}\left(Q-R \tilde{N}_{p}\right) \neq 0$ for all $R \in \mathcal{K}^{m \times n}$. Hence, we can simplify (3.11) to

$$
\mathcal{S}_{l}(G)=\left\{\left(Q-R N_{p}\right)^{-1}\left(P+R D_{p}\right): R \in \mathcal{K}^{m \times n}\right\}
$$

which is certainly nonempty. Next, $\operatorname{since} \operatorname{det}\left(Q-R \tilde{N}_{p}\right) \in$ $\mathcal{K} \backslash \mathcal{K}$, it follows as in the proof of Lemma 3.6 that every element of $\left(Q-R \tilde{N}_{p}\right)^{-1}\left(P+R \tilde{D}_{p}\right)$ is proper. Hence, $\delta_{l}(G)$ is a subset of $\mathscr{P}^{m \times n}$. Using entirely parallel reasoning, we can show that $\operatorname{det}\left(\tilde{Q}-N_{p} S\right) \in \mathcal{H} \backslash \mathscr{K}$ for every $S \in \mathcal{H}^{m \times n}$, so that

$$
S_{r}(G)=\left\{\left(\tilde{P}+D_{p} S\right)^{-1}\left(\tilde{Q}-N_{p} S\right): S \in \mathcal{H}^{n \times m}\right\} .
$$

As before, $\delta_{r}(G)$ is nonempty and is a subset of $\mathcal{P}^{m \times n}$. Note that $\delta_{l}(G) \cup \delta_{r}(G)$ is the complete set of compensators having either an rcf or an lcf that stabilizes $G$. Since this is a subset of $\mathcal{P}^{m \times n}$, the theorem is proved.

Note that, in the process of proving Theorem 4.1 we have also derived the useful characterizations (3.21) and (3.22), which do not contain the "nonsingularity" constraint found in (3.11) and (3.12).

Suppose now that $G$ is strictly proper and stable; i.e., suppose $G \in K^{n \times m}$. Then $\left(G, I_{m}\right)$ is an rcf of $G$ because (3.8) is satisfied with $P=0, Q=I_{m}$. Similarly, $\left(I_{n}, G\right)$ is an 
lcf of $G$ since (3.8) is satisfied with $\tilde{P}=0, \tilde{Q}=I_{n}$. Thus

$$
\delta_{l}(G)=\left\{\left(I_{m}-R G\right)^{-1} R: R \in \mathfrak{H}^{m \times n}\right\}
$$

is the set of all compensators in $\bigodot_{l}^{m \times n}$ that stabilize $G$, and

$$
\delta_{r}(G)=\left\{S\left(I_{n}-G S\right)^{-1}: S \in \mathcal{H}^{m \times n}\right\}
$$

is the set of all compensators in $\Theta_{r}^{m \times n}$ that stabilize $G$. The expressions (3.23) and (3.24) are generalizations of some classical results in [19] and recent results in [20]. Note that if $\mathcal{H}$ is a Bezout domain (which it is in the case of rational transfer functions), then $e_{r}^{m \times n}=e_{l}^{m \times n}=\mathscr{F}^{m \times n}$.

As an application of the general notions of properness and strict properness introduced here, we consider the stabilization of a 2-D digital filter. Let the plant $g$ be described by its transfer function

$$
g\left(z_{1}, z_{2}\right)=\sum_{i=0}^{\infty} \sum_{j=0}^{\infty} g_{i j} z_{1}^{i} z_{2}^{j}
$$

and suppose

$$
g\left(z_{1}, z_{2}\right)=n\left(z_{1}, z_{2}\right) / d\left(z_{1}, z_{2}\right)
$$

where $n$ and $d$ are polynomials without common zeros in the unit bidisk $D^{2}=\left\{\left(z_{1}, z_{2}\right):\left|z_{1}\right| \leqslant 1,\left|z_{2}\right| \leqslant 1\right\}$. Under these conditions [30] there exist polynomials $p\left(z_{1}, z_{2}\right)$ and $q\left(z_{1}, z_{2}\right)$ such that

$$
p n+q d=1, \quad \forall z_{1}, z_{2} .
$$

To apply Theorem 4.1 , let $\mathcal{H}=l_{1}\left(Z_{+}^{2}\right)$, the set of two variable power series with absolutely summable coefficient sequences, and observe that $\mathcal{H}$ contains all two variable polynomials. Thus $(n, d)$ is a coprime factorization of $g$ in $\mathcal{H}$, and it follows from Lemma 3.2 that $c$ stabilizes $g$ if and only if

$$
c\left(z_{1}, z_{2}\right)=\frac{p\left(z_{1}, z_{2}\right)+r\left(z_{1}, z_{2}\right) d\left(z_{1}, z_{2}\right)}{q\left(z_{1}, z_{2}\right)-r\left(z_{1}, z_{2}\right) n\left(z_{1}, z_{2}\right)}
$$

for some $r \in \mathcal{H}$. Now, suppose $g(. .$.$) has a zero at \left(z_{10}, z_{20}\right)$. The set

$$
\mathscr{K}=\left\{f \in \mathcal{H}: f\left(z_{10}, z_{20}\right)=0\right\}
$$

is a prime ideal in $\mathcal{H}$. Moreover, a compensator $c$ is proper if and only if $c$ does not have a pole at $\left(z_{10}, z_{20}\right)$. (Note that our assumptions on $g$ preclude $c$ having any essential singularities of the second kind in $D^{2}$.) Thus, by Theorem 4.1 , if $c$ stabilizes $g$, then $c$ does not have a pole at $\left(z_{10}, z_{20}\right)$. Since this argument can be repeated for every zero of $g$, we conclude that if $c$ stabilizes $g$, then the pole set of $c$ and the zero set of $g$ are disjoint.

\section{IV. · TOPOLOGY}

Recall that $e_{r}^{n \times m}\left(\bigodot_{l}^{n \times m}\right)$ denotes the subset of $\mathscr{F}^{n \times m}$ consisting of those $F$ that have an rcf (lcf), and $e^{n \times m}=$ $e_{r}^{n \times m} \cap \mathcal{C}_{l}^{n \times m}$ denotes the subset of $\mathscr{F}^{n \times m}$ consisting of those $F$ that have both an rcf and an lcf. Our objective in this section is to define a suitable topology on each of the sets $e_{r}^{n \times m}, e_{l}^{n \times m}$, and $e^{n \times m}$. We introduce such a topology and show that it is the weakest topology in which feedback stability is a robust property. In order to do this it is first necessary to characterize closed-loop stability in a slightly different manner from Section III.

\section{A. Closed-Loop Stability}

Consider again the system shown in Fig. 1 where $G \in$ $\mathscr{F}^{n \times m}$ and $C \in \mathscr{F}^{m \times n}$. Suppose $\operatorname{det}\left(I_{n}+G C\right) \neq 0$. Then the relation between $u_{1}, u_{2}, e_{1}, e_{2}$ can be expressed as

$$
\left[\begin{array}{l}
e_{1} \\
e_{2}
\end{array}\right]=\left[\begin{array}{cc}
\left(I_{n}+G C\right)^{-1} & -G\left(I_{m}+C G\right)^{-1} \\
C\left(I_{n}+G C\right)^{-1} & \left(I_{m}+C G\right)^{-1}
\end{array}\right]\left[\begin{array}{l}
u_{1} \\
u_{2}
\end{array}\right] .
$$

Let us denote the $(n+m) \times(n+m)$ matrix in (4.1) by $H$. Recall from Section III that the pair $(G, C)$ is stable if $\operatorname{det}\left(I_{n}+G C\right)=\operatorname{det}\left(I_{m}+C G\right) \neq 0$, and $H \in \mathcal{F}^{(n+m) \times(n+m)}$. Let us now define $R \in \mathscr{F}^{(n+m) \times(n+m)}$ and $K \in$ $\mathcal{H}^{(n+m) \times(n+m)}$ by

$$
R=\left[\begin{array}{ll}
C & 0 \\
0 & G
\end{array}\right] \quad K=\left[\begin{array}{cc}
0 & I_{n} \\
-I_{m} & 0
\end{array}\right] .
$$

One can verify that $\operatorname{det}\left(I_{n+m}+K R\right)=\operatorname{det}\left(I_{n}+G C\right)=$ $\operatorname{det}\left(I_{m}+C G\right)$. Hence, if $\operatorname{det}\left(I_{n}+G C\right) \neq 0$, then $H=\left(I_{n+m}\right.$ $+K R)^{-1}$. Thus, the pair $(G, C)$ is stable if and only if $\left(I_{n+m}+K R\right)^{-1}$ is well defined and belongs to $\mathcal{K}^{(n+m) \times(n+m)}$.

To simplify notation let $p$ denote $n+m$.

Theorem 4.1: Suppose $(G, C)$ is stable. Then $R \in e^{p \times p}$.

Remarks: The point of the theorem is that if the pair $(G, C)$ is stable, then the matrix $R$ defined by (4.2) must have both an rcf and an lcf. It would be nice if one could conclude that $G$ and $C$ individually had both an ref and an lcf, but this seems to require further investigation.

Proof: Since $(G, C)$ is stable, $H=\left(I_{p}+K R\right)^{-1} \in$ $\mathcal{K}^{p \times p}$. Note that $\operatorname{det} K=1$ so that $K^{-1} \in \mathcal{F}^{p \times p}$. Since

$$
M=R\left(I_{p}+K R\right)^{-1}=K^{-1}(I-H)
$$

we see that $M \in \mathcal{H}^{p \times p}$. Next,

$$
I_{p}-K M=I_{p}-K R\left(I_{p}+K R\right)^{-1}=\left(I_{p}+K R\right)^{-1}
$$

so that $\operatorname{det}\left(I_{p}-K M\right) \neq 0$. Also, it follows from (4.3) that $R=M\left(I_{p}-K M\right)^{-1}$. Now let $N=M, D=I_{p}-K M$. We claim that $(N, D)$ is an rcf of $R$. Clearly, $R=N D^{-1}$. Further, $(N, D)$ is right-coprime because

$$
K N+I_{p} D=K M+I_{p}-K M=I_{p} .
$$

Hence, $R \in \mathcal{C}_{r}^{p \times p}$. In the same way it can be shown that $R$ can also be written as $\left(I_{p}-M K\right)^{-1} M$, and that $\left(I_{p}-\right.$ $M K, M)$ is an lcf of $R$. Hence, $R \in \mathcal{C}_{l}^{p \times p}$.

Note that Theorem 4.1 is an abstract version of $[3$, Theorem 1]. 


\section{B. Topology}

Throughout the rest of this section we assume that $\mathcal{K}$ is in fact a topological ring; i.e., that there is a given Hausdorff topology on $\mathcal{K}$, and that addition and multiplication are continuous in this topology. We also assume that $\mathcal{F}$ (the set of units of $\mathcal{K}$ ) is an open subset of $\mathcal{K}$, and that the map $u \rightarrow u^{-1}$ of $q$ onto $q$ is continuous. ${ }^{15}$ Once we are given the topology of $\mathcal{K}$, there is a natural product topology on $\mathcal{H}^{n \times m}$. The collection of sets

$\left\{G: G=\left[\begin{array}{ccc}g_{11} & & g_{1 m} \\ \vdots & & \\ g_{n 1} & \cdots & g_{n m}\end{array}\right], \quad g_{i j} \in \Re_{i j}, \Re_{i j}\right.$ open in $\left.\mathcal{H}\right\}$

is a base for this product topology.

Recall that $e^{p \times p}$ is the set of matrices in $\mathscr{F}^{p \times p}$ that have both an rcf as well as an lcf. Now define

$$
\mathcal{S}=\left\{M \in \mathscr{F}^{p \times p}: M\left(I_{p}+K M\right)^{-1} \in \mathscr{H}^{p \times p}\right\}
$$

where $K$ is defined in (4.2). We now study the problem of defining a topology on $e^{p \times p}$. While it may be of some interest to define a topology on all of $\mathscr{F}^{p \times p}$ (and not just $e^{p \times p}$ ), we believe that $e^{p \times p}$ is a sufficiently large set for our purposes. As a matter of fact, it is actually enough to define a topology on the set of $\mathcal{S}$ (which is a subset of $\left.e^{p^{\times p}}\right)$ because Theorem 4.1 shows that a pair $(G, C)$ is stable if and only if the corresponding matrix $R$ belongs to $\delta$. However, it turns out that the topology on $\delta$ involves coprime factorizations, so that we get also a topology on $e^{p \times p}$ in the process.

Rather than define a topology on $e^{p \times p}$ arbitrarily, we postulate some requirements on the topology, and then derive the weakest possible topology that meets these requirements. ${ }^{16}$ We require that, with the given topology

$\mathrm{Rl)} \mathcal{S}$ is an open subset of $e^{p \times p}$.

R2) The function $M \rightarrow M\left(I_{p}+K M\right)^{-1}$ from $\delta$ into $\mathcal{K}^{p \times p}$ is continuous.

R3) The topology on $e^{p \times p}$ is an extension of that on $\mathfrak{H}^{p \times p}$.

Before we present the topology, a few comments are in order concerning these requirements.

1) Condition R2) means that if $(\bar{G}, \bar{C})$ is a stable pair, then $(G, C)$ is also a stable pair whenever $(G, C)$ belongs to a suitable neighborhood of $(\bar{G}, \bar{C})$; moreover, the resulting closed-loop response $H$ is "near" $\bar{H}$.

2) Since $\mathcal{H}^{p \times p}$ is a subset of $e^{p \times p}$, the desirability of condition R3) is obvious.

Lemma 4.1: Define $f: \mathcal{S} \rightarrow \mathscr{K}^{p \times p}$ by

$$
f(M)=M\left(I_{p}+K M\right)^{-1}, \quad \forall M \in \delta
$$

\footnotetext{
${ }^{15}$ The assumption that $g$ is open precludes $\mathcal{X}$ being chosen as the ring of polynomials; however, we can choose $\mathcal{H}$ as the set of power series with absolutely summable coefficient sequences.

${ }^{16}$ Given two topologies $\bar{J}_{1}$ and $\mathcal{T}_{2}$ on $\mathcal{K}^{p \times p}$, we say that $\sigma_{1}$ is weaker than $\sigma_{2}$ if $\mathscr{T}_{1}$ is a subcollection of $\sigma_{2}$.
}

and define a topology $\widetilde{T}_{1}$ on $\delta$ as follows: a subset $\delta_{1}$ of $\delta$ is open if and only if $\delta_{1}$ is the preimage under $f$ of an open subset of $\mathcal{K}^{p \times p}$. Then $\mathcal{T}_{1}$ is the weakest topology on $\delta$ in which $f$ is continuous.

The proof is omitted as it is standard [21].

Now we give an explicit description of the topology.

Definition 4.1: Define a topology $\mathcal{T}$ on $e^{p \times p}$ as follows. Given $M \in e^{p \times p}$, let $(N, D)$ be any rcf on $M$, and define a neighborhood of $M$ as the set of all ratios $N_{1} D_{1}^{-1}$, where $N_{1}, D_{1}$ belong to neighborhoods of $N$ and $D$, respectively. Define a set $\mathscr{T}$ in $e^{p \times p}$ to be open if and only if it is an arbitrary union of finite intersections of neighborhoods.

Lemma 4.2: The topology $\mathcal{T}$ of Definition 4.1 is an extension of the topology $\widetilde{T}_{1}$ on $\delta$. Also, $\mathcal{T}$ satisfies conditions R1) to R3) and is the weakest topology on $\delta$ in which $\mathrm{R} 2$ is satisfied.

Proof: The first sentence states that the topologies $\mathcal{T}$ and $\mathcal{T}_{1}$ coincide on $\delta$. We prove this by establishing two things: 1) whenever $\mathcal{X}$ is an open subset of $\mathcal{K}^{p \times p}$ and $M \in f^{-1}(\mathscr{T}), f^{-1}(\mathscr{T})$ contains a $T$-neighborhood of $M$. (This shows that $\sigma_{1}$ is weaker than $\mathcal{T}_{1}$ on $\delta$ ), and 2 ) whenever $B$ is a $\mathcal{T}$-open subset of $\mathcal{T}$ and $M \in \mathscr{B}, B$ contains the preimage under $f$ of an open subset of $\mathscr{K}^{p \times p}$ containing $f(M)$. (This shows that $\mathcal{T}$ is weaker than $\mathcal{T}_{1}$ on $\delta$.)

To prove 1), suppose $\Re$ is an open subset of $\mathcal{H}^{p \times p}$, that $M \in f^{-1}(\mathscr{N})$, and let $S=f(M)=M\left(I_{p}+K M\right)^{-1}$. We must show that there is a $T$-neighborhood $B$ of $M$, such that $f(\bar{M}) \in \Re$ whenever $\bar{M} \in \mathscr{B}$. We do this as follows. Since $M \in \mathcal{\delta}$ and $\delta$ is a subset of $\mathcal{C}, M$ has an $\operatorname{rcf}(N, D)$. Moreover, it is easy to show as in [3, Theorem 1] that $M\left(I_{p}+K M\right)^{-1} \in \mathcal{K}^{p \times p}$, if and only if $(D+K N)^{-1} \in$ $\mathcal{K}^{p \times p}$; i.e., if and only if $D+K N$ is unimodular. Finally, in such a case, $S=M\left(I_{p}+K M\right)^{-1}=N(D+K N)^{-1}$. Now by assumption the set of units $\mathcal{G}$ in $\mathcal{H}$ is open, which implies that the set of unimodular matrices $\mathcal{Q}$ in $\mathcal{K}^{p \times p}$ is open. Moreover, since the map $x \rightarrow x^{-1}$ of $g$ onto itself is assumed to be continuous, it follows that the map $U \rightarrow U^{-1}$ of $\mathcal{Q}$ onto itself is also continuous. In view of this it is immediate that there exist neighborhoods $\Re_{1}(N), \Re_{2}(D)$ such that 1) $\bar{D}+K \bar{N}$ is unimodular whenever $\bar{N} \in \Re_{1}(N)$, $\bar{D} \in \Re_{2}(D)$, and 2) $\bar{N}(\bar{D}+K \bar{N})^{-1} \in \Re$ whenever $\bar{N} \in$ $\Re_{1}(N), \bar{D} \in \Re_{2}(D)$. Thus, whenever $\bar{M}=\bar{N} \bar{D}^{-1}$ with $\bar{N} \in$ $\Re_{1}(N), \bar{D} \in \Re_{2}(D)$, we have $f(\bar{M}) \in \Re$. Thus, the required $\mathcal{T}$-neighborhood of $M$ is given by

$$
\mathscr{B}(M)=\left\{\bar{M}: \bar{M}=\bar{N} \bar{D}^{-1}, \bar{N} \in \Re_{1}(N), \bar{D} \in \Re_{2}(D)\right\} .
$$

To prove 2), suppose that $\mathscr{B}$ is a $\mathcal{T}$-open subset of $\mathcal{T}$ and that $M \in \mathscr{B}$. By definition there exists an $\operatorname{rcf}(N, D)$ of $M$ and neighborhoods $\mathscr{B}(N)$ and $\mathscr{B}(D)$ in $\mathcal{H}^{p \times p}$ such that

$$
\mathscr{B}(M)=\left\{N_{1} D_{1}^{-1}: N_{1} \in \mathscr{B}(N), D_{1} \in \mathscr{B}(D)\right\} \subset \mathscr{B} .
$$

Let $S=f(M)=M\left(I_{p}+K M\right)^{-1}$. Then $(S, I-K S)$ is an rcf of $M$. Moreover, by [8, p. 401, Property 2] there exists a $P \in \mathcal{H}^{p \times p}$ such that $P^{-1} \in \mathcal{H}^{p \times p}$, and $N=S P, D=\left(I_{p}-\right.$ 
$K S) P$. Now find a neighborhood $\mathscr{B}(S)$ in $\mathcal{K}^{p \times p}$ such that $\Re(S) P \subset \Re(N),\left(I_{p}-K \Re(S)\right) P \subset \Re(D)$. Then

$$
\begin{aligned}
f^{-1}(\Re(S)) & =\left\{\bar{S}\left(I_{p}-K \bar{S}\right)^{-1}, \forall \bar{S} \in \Re(S)\right\} \\
& =\left\{\bar{S} P\left[\left(I_{p}-K \bar{S}\right) P\right]^{-1}, \forall \bar{S} \in \mathscr{B}(S)\right\} \\
& \subset \Re(M) \subset \mathscr{B} .
\end{aligned}
$$

Next it follows from Lemma 4.1 that $\sigma$ satisfies conditions R1) and R2), and that $\mathcal{G}$ is the weakest topology on $\mathcal{S}$ satisfying R2). To prove that the $\sigma_{\text {-topology on }} e^{p \times p}$ (viewed as a subset of $\mathcal{H}^{p \times p}$ ) is the same as the original topology on $\mathcal{H}^{p \times p}$, we observe that if $H \in \mathcal{H}^{p \times p}$, then $\left(H, I_{p}\right)$ is an ref of $H$. The rest of the argument is left to the reader.

Up to now we have defined a topology on the set $\mathcal{C}^{p \times p}$ where $p=n+m$, and have shown that it is the weakest topology on $e^{p \times p}$ such that the map $M \rightarrow M\left(I_{p}+K M\right)^{-1}$ is continuous wherever it is defined. However, what we are really interested in is a topology on the subsystems $G$ and $C$ and not just a topology on the set of matrices $R=$ $\operatorname{diag}\{C, G\}$.

We begin by observing that we can define the topology of Definition 4.1 on a set $e^{n \times m}$ even if $n \neq m$. Let us label this topology as $\widetilde{\sigma}$; then it is clear what we mean by the topological spaces $\left(e^{n \times m}, \mathcal{T}\right)$ and $\left(e^{m \times n}, \sigma\right)$.

Next let us define

$$
\Re=\left\{R=\left[\begin{array}{ll}
C & 0 \\
0 & G
\end{array}\right], C \in e^{m \times n}, G \in e^{n \times m}\right\} .
$$

Lemma 4.3: $\Re$ is a subset of $e^{p \times p}$.

Proof: Let $\left(N_{G}, D_{G}\right),\left(N_{C}, D_{C}\right)$ be rcf's of $G$ and $C$, and let $\left(\tilde{D}_{G}, \tilde{N}_{G}\right),\left(\tilde{D}_{C}, \tilde{N}_{C}\right)$ be lcf's of $G$ and $C$. Then

$$
\left(\left[\begin{array}{cc}
N_{C} & 0 \\
0 & N_{G}
\end{array}\right],\left[\begin{array}{cc}
D_{C} & \\
0 & D_{G}
\end{array}\right]\right),\left(\left[\begin{array}{cc}
\tilde{D}_{C} & 0 \\
0 & \tilde{D}_{G}
\end{array}\right],\left[\begin{array}{cc}
\tilde{N}_{C} & 0 \\
0 & \tilde{N}_{G}
\end{array}\right]\right)
$$

are an rcf and an lcf of $R$. Hence, $R \subset e^{p \times p}$.

The set $\Re$ can be identified with the Cartesian product $e^{m \times n} \times e^{n \times m}$ in an obvious way and can be given the product topology. In this product topology a neighborhood of $R \in R$ is given by

$$
\mathscr{B}(R)=\left\{\bar{R}=\left[\begin{array}{ll}
\bar{C} & 0 \\
0 & \bar{G}
\end{array}\right], \bar{C} \in \mathscr{B}(C), \bar{G} \in \mathfrak{B}(G)\right\}
$$

where $\mathscr{B}(C)$ and $\mathscr{B}(G)$ are neighborhoods of $C$ and $G$ in $\left(e^{m \times n}, \sigma\right)$ and $\left(e^{n \times m}, \sigma\right)$, respectively. In view of the proof of Lemma 4.3, the next result is obvious.

Lemma 4.4: The product topology on $\mathcal{R}$ is the same as its relative topology obtained by viewing $\Re$ as a subset of $e^{p \times p}$.

We are now in a position to combine Lemmas 4.2 and 4.4 , and state the main result of this section.
Theorem 4.1: Consider the system of Fig. 1, where $C \in$ $e^{m \times n}, G \in e^{n \times m}$

Under these conditions

1) if we equip $\mathcal{C}^{m \times n}$ and $\mathcal{C}^{n \times m}$ with the topology of Definition 4.1, then the map $(C, G) \rightarrow H$ [cf (4.1)] is continuous wherever it is defined, and

2) the topology of Definition 4.1 on the product space is the weakest topology such that the map $(C, G) \rightarrow H$ is continuous wherever it is defined.

Theorem 4.1 implies that if we wish to design a stabilizing compensator for a plant $G$, we must know approximately an $\mathrm{rcf}$ of $G$. Suppose we are given a nominal plant description $\bar{G}$, based on which we design a nominal compensator $\bar{C}$. Then the actual system will continue to be stable as long as the actual $G$ and $C$ are sufficiently closed to $\bar{G}$ and $\bar{C}$ in the sense of the topology of Definition 4.1, and moreover, the actual closed-loop transfer matrix $H$ will be close to its nominal value.

\section{Properties of the Topology .}

In this subsection we explore some of the properties of the topology defined in Section IV-B. In particular, we show that one can use either rcf's or lcf's in defining the topology, and that multiplication and division are not necessarily continuous in this topology.

In Definition $4.1^{17}$ a neighborhood of $M \in e^{n \times m}$ is defined to be the set of all ratios $N_{1} D_{1}^{-1}$, where $N_{1}, D_{1}$ are in some neighborhoods of $N$ and $D$, and $(N, D)$ is an ref of $M$. The choice of an rcf in Definition 4.1 (rather than an lcf) may appear to be arbitrary. We can define an alternate topology on $e^{n \times m}$ as follows: given $M \in \mathrm{e}^{n \times m}$, a left neighborhood of $M$ is the set of all ratios $\tilde{D}_{1}^{-1} \tilde{N}_{1}$, where $\tilde{N}_{1}, \tilde{D}_{1}$ belong to neighborhoods of $\tilde{N}, \tilde{D}$, and $(\tilde{D}, \tilde{N})$ is an lcf of $M$. The left topology on $e^{n \times m}$ consists of the collection of arbitrary unions of finite intersections of left neighborhoods. The objective now is to show that the left topology on $e^{n \times m}$ coincides with that of Definition 4.1. In order to do this, we require the concept of a doubly coprime fàctorization, introduced in [7], [22].

Lemma 4.5 [7], [22]: Suppose $M \in \mathrm{e}^{n \times m}$, and let $(N, D),(\tilde{D}, \tilde{N})$ be any rcf and lcf of $M$. Then there exist $P \in \mathcal{H}^{m \times n}, Q \in \mathcal{H}^{m \times m}, P \tilde{P} \in \mathcal{H}^{m \times n}$, and $\tilde{Q} \in \mathcal{H}^{n \times n}$ such that

$$
\begin{aligned}
P N+Q D & =I_{m} \\
\tilde{N} \tilde{P}+\tilde{D} \tilde{Q} & =I_{n} \\
P \tilde{Q} & =Q \tilde{P} .
\end{aligned}
$$

Proof: Given the pair $(N, D)$ and $(\tilde{D}, \tilde{N})$, find $P, Q, \tilde{P}_{1}, \tilde{Q}_{1}$ such that

$$
\begin{gathered}
P N+Q D=I_{m} \\
\tilde{N} \tilde{P}_{1}+\tilde{D} \tilde{Q}_{1}=I_{n} .
\end{gathered}
$$

Now define

\footnotetext{
${ }^{17}$ Actually, Definition 4.1 is stated for the case $n=m=p$, but the extension to the case $n \neq m$ is obvious.
} 


$$
E=\left[\begin{array}{cc}
Q & P \\
-\tilde{N} & \tilde{D}
\end{array}\right]
$$

and observe that

$$
E \cdot\left[\begin{array}{cc}
D & -\tilde{P}_{1} \\
N & \tilde{Q}_{1}
\end{array}\right]=\left[\begin{array}{cc}
I_{m} & -Q \tilde{P}_{1}+P \tilde{Q}_{1} \\
0 & I_{m}
\end{array}\right] \triangleq U .
$$

Since $\operatorname{det} U=1$ from (4.18), and since $E$ is a left divisor of the unimodular matrix $U$, it follows that $E$ is itself unimodular. Let

$$
\left[\begin{array}{r}
-\tilde{P} \\
\tilde{Q}
\end{array}\right]=E^{-1}\left[\begin{array}{c}
0 \\
I_{n}
\end{array}\right]
$$

Then it follows from (4.18) and (4.19) that

$$
E\left[\begin{array}{rr}
D & -\tilde{P} \\
N & \tilde{Q}
\end{array}\right]=I_{n+m}
$$

which implies (4.15).

The next result shows that the topology of Definition 4.1 (which may be called the right topology) and the left topology on $e^{p \times p}$ coincide. Strictly speaking it only proves that the right topology on $e^{p \times p}$ is stronger than the left topology on $e^{p \times p}$. However, the converse is proved in an entirely analogous fashion.

Lemma 4.6: Suppose $M \in e^{p \times p}$, and let $(N, D),(D, N)$ be any rcf and lcf of $M$. Under these conditions there exist neighborhoods $\mathscr{B}(N), \mathscr{B}(D)$ in $\mathfrak{K}^{p \times p}$ such that, whenever $N_{1} \in \mathscr{B}(N)$ and $D_{1} \in \mathscr{B}(D), \quad M_{1}=N_{1} D_{1}^{-1}$ has an lef $\left(D_{1}\left(N_{1}, D_{1}\right), \tilde{N}_{1}\left(N_{1}, D_{1}\right)\right)$ such that $\tilde{D}_{1}$ and $\tilde{N}_{1}$ are continuous functions of $\left(N_{1}, D_{1}\right)$, and such that $\tilde{D}_{1}(N, D)=\tilde{D}$, $\tilde{N}_{\mathrm{l}}(N, D)=\tilde{N}$.

Proof: Given $(N, D),(\tilde{D}, \tilde{N})$, select $P, Q, \tilde{P}, \tilde{Q}$ to satisfy (4.15). Now consider the matrix

$$
C_{1}\left(N_{1}, D_{1}\right)=\left[\begin{array}{rr}
D_{1} & -\tilde{P} \\
N_{1} & \tilde{Q}
\end{array}\right] .
$$

Note that $\operatorname{det} C_{1}(N, D) \in \mathcal{g}$. Since $\mathcal{g}$ is an open set and since the determinant of a matrix is a continuous function of its elements, there exist neighborhoods $\mathscr{B}(N), \mathscr{B}(D)$ such that $\operatorname{det} C_{1}\left(N_{1}, D_{1}\right) \in \mathcal{g}$ whenever $N_{1} \in \mathscr{B}(N), D_{1} \in$ $\mathscr{B}(D)$. Let $E\left(N_{1}, D_{1}\right)=\left[C_{1}\left(N_{1}, D_{1}\right)\right]^{-1}$, and partition this inverse as

$$
E\left(N_{1}, D_{1}\right)=\left[\begin{array}{rr}
Q_{1} & P_{1} \\
-\tilde{N}_{1} & -\tilde{D}_{1}
\end{array}\right]
$$

where all elements are continuous functions of $\left(N_{1}, D_{1}\right)$. Then $\tilde{D}_{1}, \tilde{N}_{1}$ are the required functions.

Now that the topology on $e^{n \times m}$ has been defined, we examine what convergence in this topology means. Recall that a sequence $\left\{M_{i}\right\}$ in $e^{n \times m}$ converges to $M \in e^{n \times m}$ if and only if every neighborhood of $M$ contains all but a finite number of terms in the sequence $\left\{M_{i}\right\}$. Thus, applying Definition 4.1 we see that a sequence $\left\{M_{i}\right\}$ in $e^{n \times m}$ converges to $M \in e^{n \times m}$ if and only if the following is true: given any $\operatorname{rcf}(N, D)$ of $N$, there exist rcf's $\left(N_{i}, D_{i}\right)$ of $M_{i}$ such that $N_{i} \rightarrow N$ in $\mathcal{H}^{n \times m}, D_{i} \rightarrow D$ in $\mathcal{H}^{m \times n}$.

However, a further simplification is possible if we make use of the uniqueness of rcf's to within a unimodular factor.

Lemma 4.7: A sequence $\left\{M_{i}\right\}$ in $e^{n \times m}$ converges to $M \in e^{n \times m}$ if and only if either of the following equivalent statements is true.

1) There exists an $\operatorname{rcf}(\bar{N}, \bar{D})$ of $M$ and right fractional representations $\left(\bar{N}_{i}, \bar{D}_{i}\right)$ of $M_{i}$ such that $\bar{N}_{i} \rightarrow N$ in $\mathcal{Y}^{m \times m}$ and $\bar{D}_{i} \rightarrow \bar{D}$ in $\mathcal{H}^{m \times m}$.

2) There exists an lcf $(\tilde{D}, \tilde{N})$ of $M$ and left fractional representations $\left(\tilde{D}_{i}, \tilde{N}_{i}\right)$ of $M_{i}$ such that $\tilde{N}_{i} \rightarrow \tilde{N}$ in $\mathcal{H}^{n \times m}$ and $\tilde{D}_{i} \rightarrow \tilde{D}$ in $\mathcal{H}^{n \times n}$.

Proof: We only prove that convergence in $e^{n \times m}$ is equivalent to 1 ); the equivalence of 1 ) and 2) follows from Lemma 4.6.

We begin by establishing that if $\bar{N}_{i} \rightarrow \bar{N}, \bar{D}_{i} \rightarrow \bar{D}$ and $(\bar{N}, \bar{D})$ is right-coprime, then $\left(\bar{N}_{i}, \bar{D}_{i}\right)$ is also right-coprime for sufficiently large $i$ so that $\bar{N}_{i}, \bar{D}_{i}$ ) is actually an ref of $M_{i}$ for sufficiently large $i$. Select $P \in \mathfrak{F}^{m \times n}, Q \in \mathfrak{H}^{m \times m}$ such that

$$
P \bar{N}+Q \bar{D}=I_{m}
$$

Then

$$
P \bar{N}_{i}+Q \bar{D}_{i}=I_{m}+P\left(\bar{N}_{i}-\bar{N}\right)+Q\left(\bar{D}_{i}-\bar{D}\right)=F_{i}, \quad \text { say. }
$$

Since addition and multiplication on $\mathscr{H}$ are continuous and since $\mathcal{G}$ is an open subset of $\mathcal{K}$, we see that det $F_{i} \in \mathcal{G}$ for sufficiently large $i$; i.e., that $F_{i}^{-1} \in \mathcal{T}^{m \times n}$ for sufficiently large $i$. Hence

$$
F_{i}^{-1} P \bar{N}_{i}+F_{i}^{-1} Q \bar{D}_{i}=I_{m} \quad \text { for sufficiently large } i
$$

so that $\left(\bar{N}_{i}, \bar{D}_{i}\right)$ is right-coprime for sufficiently large $i$. Since a finite number of terms from a sequence can be discarded without effecting its limit, we can assume without loss of generality that $\left(\bar{N}_{i}, \bar{D}_{i}\right)$ is an ref of $M_{i}$.

It is clear that if $\left\{M_{i}\right\}$ converges to $M$ in $e^{n \times m}$, then 1) is true. To prove the reverse implication, let $(N, D)$ be any rcf of $M$. Then there exists a $P \in \mathscr{g}_{m}$ such that $N=\bar{N} P, D=\bar{D} P$; also $\left(\bar{N}_{i} P, \bar{D}_{i} P\right)$ is an rcf of $M_{i}$. Since multiplication on $\mathcal{H}^{m \times m}$ is continuous, $\bar{N}_{i} P \rightarrow \bar{N} P=N, D_{i} P \rightarrow \bar{D} P=D$ as $i \rightarrow \infty$. Thus $\left\{M_{i}\right\}$ converges to $M$.

We conclude this section by studying some properties of the map $(N, D) \rightarrow N D^{-1}$. To keep the exposition simple we restrict ourselves to the scalar case, but the extensions to the matrix case are readily apparent. Thus, let $\mathscr{F}$ denote the quotient field of $\mathcal{K}$, and let $\mathcal{C}$ denote the subset of $\mathscr{F}$ consisting of those elements that have coprime factorizations.

Definition 4.2: We define $\pi: \mathscr{H} x(\mathcal{H} \backslash 0) \rightarrow \mathscr{F}$ by $\pi(n, d)$ $=n / d$.

Lemma 4.8: Suppose $\mathscr{U}$ is an open subset of $\mathscr{H} x(\mathfrak{H} \backslash 0)$, and that $\pi(\mathscr{U}) \subseteq \mathcal{C}$. Then $\pi(\mathscr{U})$ is open in $\mathrm{C}$. 
Proof: Since the subbase for the topology on $\mathcal{C}$ consists of images under $\pi$ of open sets in $\mathcal{F} x(\mathcal{F} \backslash 0)$, result follows immediately.

Lemma 4.9: In general, $\pi$ is not a continuous map.

Proof: We show this by example. Let $\mathcal{K}=\mathscr{Q}$, the Banach algebra of Example 2.2, and let $\mathscr{F}$ denote the quotient field of $\mathcal{H}$. Let $\mathcal{C}$ denote the subset of $\mathscr{F}$ consisting of elements that have a coprime factorization in $\mathcal{X}$. For the sake of convenience we represent elements of $\mathcal{H}$ by their Laplace transforms. Now suppose

$$
\hat{n}_{\epsilon}(s)=\frac{s-1+\epsilon}{s+1}, \quad \hat{d}_{\epsilon}(s)=\frac{s-1}{s+1} .
$$

Then

$$
\begin{aligned}
& \pi\left(\hat{n}_{\epsilon}, \hat{d}_{\epsilon}\right) \triangleq \hat{g}_{\epsilon}(s)=\frac{s-1+\epsilon}{s-1} \\
& \pi\left(\hat{n}_{0}, \hat{d}_{0}\right) \triangleq \hat{g}_{0}(s)=1 .
\end{aligned}
$$

As can be easily verified, $\hat{g}_{\epsilon} \in \mathcal{C}$ for all $\epsilon$. Now, as $\epsilon \rightarrow 0$, $\hat{n}_{\mathrm{c}} \rightarrow \hat{n}_{0}$ and $\hat{d}_{\mathrm{c}} \rightarrow \hat{d}_{0}$ in the topology of $\mathcal{H}$. However, we claim that $\hat{g}_{\varepsilon}$ does not converge to $\hat{g}_{0}$ in the topology on $\mathcal{C}$. To see this, observe that $(1,1)$ is an rcf of $\hat{g}_{0}$, and define the ball $B(1,1)$ as the ball of elements whose distance from the unit element of $\mathcal{H}$ is less than one; note that every element of $B(1,1)$ is invertible, i.e., $B(1,1) \subseteq g$. Now we claim that whenever $\epsilon \neq 0, \hat{g}_{\epsilon}$ does not have a coprime factorization $\left(\hat{n}_{\mathfrak{c}}, \hat{d}_{\mathrm{f}}\right)$ with $\hat{n}_{\mathrm{c}} \in B(1,1), \hat{d}_{\mathrm{c}} \in B(1,1), \hat{d}_{\mathrm{c}} \in B(1,1)$; if it did, then $\hat{d}_{\mathrm{\varepsilon}} \in \mathcal{G}$ implies that $\hat{g}_{\mathrm{\varepsilon}}=\hat{n}_{\mathrm{e}} \cdot \hat{d}_{\mathrm{c}}^{-1} \in \mathcal{H}$, and clearly $\hat{g}_{\mathrm{c}} \notin \mathcal{H}$ whenever $\epsilon \neq 0$.

Since the mapping $\pi$ is not, in general, continuous, it follows that addition and multiplication on the set $e$ are not, in general, continuous; i.e., $e$ cannot, in general, be imbedded in a topological ring. However, this observation should not be too surprising. Every engineer knows that a plant with an imperfect pole-zero cancellation (such as $\hat{g}_{\epsilon}$ ) may not be stabilized by a controller that stabilizes a plant with a perfect pole-zero cancellation (such as $\hat{g}_{0}$ ). Thus, there is no reason to expect $\hat{g}_{c}$ to converge to $\hat{g}_{0}$. On the other hand, note that whenever $\delta \neq 0$, the family

$$
g_{\epsilon, \delta}(s)=\frac{s-1-\epsilon}{s-1-\delta}
$$

converges to

$$
\hat{g}_{0, \delta}(s)=\frac{s-1}{s-1-\delta}
$$

as $\epsilon \rightarrow 0$, in the chosen topology on $e$. This, too, is reasonable because both $\hat{g}_{\epsilon, \delta}$ and $\hat{g}_{0, \delta}$ have imperfect pole-zero cancellations, which would be stabilized in the same manner.

\section{Conclusions}

In this paper we have given fairly complete results concerning various algebraic and topological aspects of feedback stabilization. These results extend in a significant way the previously known results on this subject and appear to present some promising avenues for future research. In particular, it is of interest to determine various classes of plants for which stabilizing compensators can be shown to exist. One can also investigate feedback stabilization in the case where the outputs to be regulated are not necessarily the outputs available to be fed back. This is done for the rational case in [23], [24]. Another open problem is to show that both $G$ and $F$ must have both ref's and lcf's in order for the feedback system (4.1) to be stable.

An important special case of the results given here arises when $\mathcal{H}$ is the set of proper stable rational functions. This case is studied in detail [25].

One of the useful applications of a topology for unstable plants is to the robust servo problem. This is studied in [25].

Much of the contents of Sections III and. IV can be extended to encompass nonlinear systems, in which case $\mathcal{H}$ is not a ring, but is left-distributive algebra. This is done in [26].

In this paper we represent linear systems by their input-output map without worrying about the internal (e.g., state variable) representation. Such representations might not exist for the general class of systems studied here.

\section{REFERENCES}

[1] N. Jacobson, Lectures in Abstract Algebra, vol. 1. New York: Van Nostrand, 1953.

[2] M. Vidyasagar, "Input-output stability of a broad class of linear time-invariant multivariable feedback systems," SIAM J. Contr., vol. 10, pp. 203-209, Feb. 1972.

[3] M. Vidyasagar, "Coprime factorizations and the stability of multivariable distributed feedback systems," SIAM J. Contr., vol. 13, pp. 1144-1155, Nov. 1975.

[4] M. Vidyasagar, "On the use of right-coprime factorizations in distributed feedback systems containing unstable subsystems," IEEE Trans. Circuits Syst., vol. CAS-25, pp. 916-921, Nov. 1978.

[5] N. T. Hung and B. D. O. Anderson, "Triangularization technique for the design of multivariable control systems," IEEE Trans. A utomat. Contr, vol. AC-24, pp. 455-460, June 1979.

[6] F. M. Callier and C. A. Desoer, "An algebra of transfer functions of distributed linear time-invariant systems," IEEE Trans. Circuits Syst., yol. CAS-25, pp. 651-662, Sept. 1978.

[7] F. M. Callier and C. A. Desoer, "Stabilization tracking and disturbance rejection in multivariable convolution systems," Univ. California, Electron. Res. Lab. Rep. UCB/ERI M78/83, Der. 1978.

[8] C. A. Desoer, R.-W. Liu, J. Murray, and R. Saeks, "Feedback system design: The fractional approach to analysis and synthesis," IEEE Truns. Automat. Contr., vol. AC-25, pp. 399-412, June 1980

[9] D. C. Youla, H. A. Jabr, and J. J. Bongiono, Jr., "Modern Wiener-Hopf design of optimal controllers, Part II: The multivariable case," IEEE Trans. Automat. Contr., vol. AC-21, pp. 319-338, June 1976.

[10] G. Bengtsson, "Output regulation and internal models: A frequency domain approach," Automatica, vol. 13, pp. 333-345, 1977.

[1I] L. Pernebo, "An algebraic theory for the design of controllers for linear multivariable systems," IEEE Truns. Automat. Contr., vol. AC-26, pp. 171-194, Feb. 1981.

[12] C. C. MacDuffee, Theory of Matrices. New York: Chelsea, 1956, p. 35 .

[13] C. A. Desoer and M. Vidyasagar, Feedback Systems: Input-Output Properties. New York: Academic, 1975.

[14] E. Hille and R. S. Phillips, Functional Analysis and Semigroups. Providence, RI: Amer. Math. Soc., 1957.

[15] M. Vidyasagar and N. K. Bose, "Input-output stability of linear systems defined over measure spaces," in Proc. Midwest Symp. Circ. Syst., Montreal, P.Q., Canada, Aug. 1975, pp. 394-397.

[16] W. Rudin, Real and Complex Anulysis, 2nd ed. New York: McGraw-Hill, 1974.

[17] F. M. Callier and C. A. Desoer, "Open-loop unstable convolution feedback systems with dynamical feedback," Automaticu, vol. 13, pp. 507-518, Dec. 1976. 
[18] B. A. Francis, "The multivariable servomechanism problem from the input-output viewpoint," IEEE Trans. Automat. Contr., vol. AC-22, pp. 322-328, June 1977 .

[19] G. C. Newton, L. A. Gould, and J. F. Kaiser, Analytic Design of Linear Feedback Controls. New York: Wiley, 1957.

[20] G. Zames, "Feedback and optimal sensitivity: Model reference transformations, weighted seminorms, and approximate universes," IEEE Trans. Automat. Contr., vol. AC-26, pp. 301-320, Apr. 1981.

(21] J. L. Kelley, General Topology. New York: Van Nostrand, 1955, p. 94.

[22] R. Saeks and J. W. Murray, "Feedback system design: The tracking and disturbance rejection problems," IEEE Trans. Automat. Contr., vol. AC-26, pp. 203-218, Feb. 1981.

[23] L. Cheng and J. B. Pearson, "Frequency domain synthesis of multivariable linear regulators," IEEE Trans. Automat. Contr., vol. AC-23, pp. 3-15, Feb. 1978.

[24] L. Cheng and J. B. Pearson, "Synthesis of linear-multivariable regulators," IEEE Trans. Automat. Contr., vol. AC-26, pp. 194-202, Feb. 1981 .

[25] B. A. Francis and M. Vidyasagar, "Algebraic and Topological aspects of the servo problem for lumped linear systems," Dep. Eng. Appl. Sci., Yale Univ., New Haven, CT, S \& IS Rep. 8003, 1980.

[26] M. Vidyasagar, "A topology for unstable nonlinear systems and robustness of feedback stability," in preparation.

[27] O. Zariski and P. Samuel, Commutative Algebra, vol. I. New York: Van Nostrand, 1953.

[28] M. Vidyasagar, H. Schneider, and B. A. Francis, "Algebraic and topological aspects of feedback stabilization," Dep. Elec. Eng., Univ. Waterloo, Waterloo, Ont., Canada, Rep. $8009,1980$.

[29] K. Hoffman, Banach Spaces of Analytic Functions. Englewood Cliffs, NJ: Prentice-Hall, 1962.

[30] M. F. Atiyah and I. G. MacDonald, Introduction to Commutative Algebra. Reading, MA: Addison-Wesley, 1969.

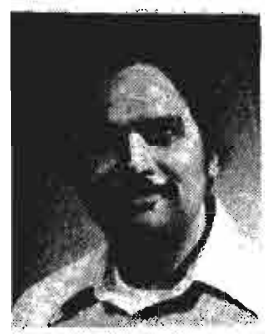

Mathukumalli Vidyasagar (S'69-M'69-M'77SM'78) was born in Guntur, Andhra Pradesh, India, on September 29, 1947. He received the B.S., M.S., and Ph.D. degrees, all in electrical engineering, from the University of Wisconsin, Madison, in 1965, 1967, and 1969, respectively.

After completing the Ph.D. degree, he taught for one year at Marquette University, Milwaukee, WI. During the period 1970-1980 he was with Concordia University, Montreal, P.Q. Canada. Since 1980 he has been with the Department of Electrical Engineering at the University of Waterloo, Waterloo, Ont., Canada. During the academic year 1972-1973 he was a Visiting Assistant Professor with the Department of System Science, University of California, Los Angeles, and during the Summer of 1973 he was a Visiting Assistant Research Engineer with the Electronics Research Laboratory, University of California, Berkeley. He has authored several technical papers, and is coauthor of Feedback Systems: Input-Output Properties (Academic, 1975), the author of Nonlinear Systems Analysis (Prentice-Hall, 1978) and Input -Output Analysis of Large-Scale Interconnected Systems (Springer-Verlag, 1981), and a coauthor of Nonlinear Systems: Stability Analysis (Dowden, Hutchinson and Ross, 1977). His current research interests are algebraic system theory, stability theory, and multidimensional systems.

Dr. Vidyasagar was awarded the E.W.R. Steacie Memorial Fellowship by the Natural Sciences and Engineering Research Council of Canada for the period 1981-1983. He was an Associate Editor of IEEE TRANSAC- tions on Automatic Control, and is a member of the Technical Committee on Nonlinear Circuits and Systems of the IEEE Circuits and Systems Society.

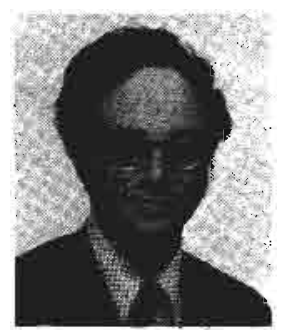

Hans Schneider was born in Vienna, Austria, on January 24, 1927. He received the M.A. and Ph.D. degrees from the University of Edinburgh, Edinburgh, Scotland, in 1948 and 1952, respectively.

From 1952 to 1959 he was a Lecturer at the Queen's University of Belfast, Belfast, Ireland and since 1959 he has held a position at the University of Wisconsin, Madison, WI, where he was Department of Mathematics Chairman from 1966 to 1968. He has also been a Visiting Professor at Washington State University, Pullman, the University of Cajifornia, Santa Barbara, the University of Toronto, Toronto, Ont., Canada, the Technical University of Munich, Munich, Germany, Centre de Recherches Mathématiques, Montreal, P.Q., Canada, and the University of Würzburg, Würzburg, Germany. He is the author of over 60 research papers and two undergraduate textbooks. Many of his publications are joint. His main field of specialization is matrix theory in its various forms. He has made contributions to the theory of nonnegative matrices (Perron-Frobenius theory) and to generalization to operators leaving a cone invariant. He has worked on $M$-matrices, norms, and numerical ranges of matrices, and on inertia (Lyapunov) theory. Recently, he has studied scaling problems of matrices and associated graph theoretic problems. $\mathrm{He}$ has also made some contributions to semigroups and universal algebras. Eleven students have obtained Ph.D's under his direction.

Dr. Schneider is Editor-in-Chief of Linear Algebra and its Applications, Advisory Editor of Letters in Linear Algebra, and an Editor of Linear and Multilinear Algebra and the SIAM Journal of Algebraic and Discrete Methods.

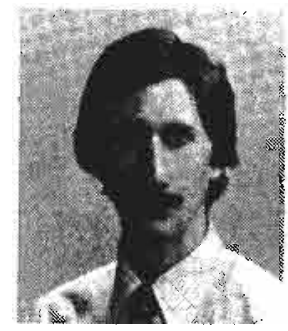

Bruce A. Francis (S'73-M'75) was born in Toronto, Ont., Canada, on October 8,1947 . He received the B.A.Sc. and M.Eng. degrees in mechanical engineering and the Ph.D. degree in electrical engineering from the University of Toronto in 1969, 1970, and 1975, respectively.

He was awarded a National Research Council of Canada Postdoctoral Fellowship during 19751977, which he held at the University of California, Berkeley, and at the University of Cambridge, Cambridge, England. During 1977-1979 he was with the Department of Electrical Engineering, McGill University, Montreal, P.Q., Canada and during 1979-1981 he was with the Department of Engineering and Applied Science, Yale University, New Haven, CT. Currently, he is an Associate Professor in the Department of Electrical Engineering at the University of Waterloo, Waterloo, Ont., Canada. $\mathrm{He}$ has also held a visiting research position at Concordia University, Montreal.

Dr. Francis was an Associate Editor for the IEEE TRANSACTIONS on Automatic CONTROL during 1979-1980. He is a member of AMS and SIAM. 